\title{
Toughness, Forbidden Subgraphs and Pancyclicity
}

\author{
Wei Zheng ${ }^{1,2,3} \cdot$ Hajo Broersma ${ }^{2}$ (D) Ligong Wang ${ }^{3}$
}

Received: 22 May 2019/Revised: 15 January 2021 / Accepted: 27 January 2021 /

Published online: 19 February 2021

(C) The Author(s) 2021

\begin{abstract}
Motivated by several conjectures due to Nikoghosyan, in a recent article due to $\mathrm{Li}$ et al., the aim was to characterize all possible graphs $H$ such that every 1-tough $H$ free graph is hamiltonian. The almost complete answer was given there by the conclusion that every proper induced subgraph $H$ of $K_{1} \cup P_{4}$ can act as a forbidden subgraph to ensure that every 1-tough $H$-free graph is hamiltonian, and that there is no other forbidden subgraph with this property, except possibly for the graph $K_{1} \cup$ $P_{4}$ itself. The hamiltonicity of 1-tough $K_{1} \cup P_{4}$-free graphs, as conjectured by Nikoghosyan, was left there as an open case. In this paper, we consider the stronger property of pancyclicity under the same condition. We find that the results are completely analogous to the hamiltonian case: every graph $H$ such that any 1-tough $H$-free graph is hamiltonian also ensures that every 1-tough $H$-free graph is pancyclic, except for a few specific classes of graphs. Moreover, there is no other forbidden subgraph having this property. With respect to the open case for hamiltonicity of 1-tough $K_{1} \cup P_{4}$-free graphs we give infinite families of graphs that are not pancyclic.
\end{abstract}

Keywords Toughness · Forbidden subgraph · Pancyclic graph · Hamiltonian graph

Mathematics Subject Classification 05C38 -05C42 - 05C45

\section{Introduction}

In this paper, we consider only undirected, finite and simple graphs. The terminology and notation not defined here can be found in [6].

Supported by the National Natural Science Foundation of China (no. 11871398), the Natural Science Basic Research Plan in Shaanxi Province of China (no. 2018JM1032) and the China Scholarship Council (no. 201706290168)

Extended author information available on the last page of the article 
Let $G$ be a graph with vertex set $V(G)$ and edge set $E(G)$. For a vertex $u \in V(G)$ and a subgraph $H$ of $G$, the neighborhood of $u$ in $H$ is denoted by $N_{H}(u)=\{v \in V(H) \mid u v \in E(G)\}$, and the degree of $u$ in $H$ is denoted by $d_{H}(u)=\left|N_{H}(u)\right|$. The length of a path or cycle is its number of edges. For two vertices $u$ and $v$ in a connected graph $H$, the distance between $u$ and $v$ in $H$, denoted by $d_{H}(u, v)$, is the length of a shortest $(u, v)$-path in $H$, which is a path connecting $u$ and $v$. When there is no danger of ambiguity, we use $N(u), d(u)$ and $d(u, v)$ instead of $N_{G}(u), d_{G}(u)$ and $d_{G}(u, v)$, respectively. The girth of a graph $G$ is the length of a shortest cycle of $G$, and the circumference is the length of a longest cycle of $G$. The complete bipartite graph on $m+n$ vertices, denoted by $K_{m, n}$, consists of a vertex set $A \cup B$ with $|A|=m>0, \quad|B|=n>0$, and $A \cap B=\emptyset$, and the edge set $\{u v \mid u \in A, v \in B\}$. We say that $K_{m, n}$ is balanced if $m=n$. For a subset $M$ of $E(G)$, we say $M$ is a matching of $G$ if no two edges of $M$ share an end vertex, and we say a matching $M$ is perfect if $V(M)=V(G)$. We use $|M|$ to denote the number of edges of $M$.

Let $\omega(G)$ denote the number of components of the graph $G$. As introduced in [11], a connected graph $G$ is said to be $t$-tough if $t \cdot \omega(G-X) \leq|X|$ for all $X \subseteq$ $V(G)$ with $\omega(G-X)>1$. The toughness of $G$, denoted $\tau(G)$, is the maximum value of $t$ such that $G$ is $t$-tough (taking $\tau\left(K_{n}\right)=\infty$ for all $n \geq 1$ ). For a subset $S$ of $V(G)$, we use $\langle S\rangle$ to denote the subgraph of $G$ induced by $S$. If the subgraph induced by $S$ is isomorphic to a graph $H$, we also write $S \cong H$. For a given graph $H$, we say $G$ is $H$ free if $G$ does not contain an induced copy of $H$.

A cycle (path) in a graph $G$ is called a Hamilton cycle (Hamilton path) if it contains all vertices of $G$, and $G$ is called hamiltonian if it contains a Hamilton cycle. $G$ is called hamiltonian-connected if there is a Hamilton path between any two distinct vertices of $G$. A graph of order $n$ is called pancyclic if it contains cycles of any length from 3 up to $n$. Obviously, both hamiltonian-connected graphs (on $n \geq 3$ vertices) and pancyclic graphs are hamiltonian graphs. A lot of research has been devoted to these hamiltonian properties, and it has been observed that various sufficient conditions for a graph to be hamiltonian are so strong that they imply considerably more about the cycle structure of the graph. Based on this observation, Bondy [5] presented a metaconjecture in 1971 in which he stated that almost any nontrivial condition on a graph which implies that the graph is hamiltonian also implies that it is pancyclic (except possibly for a simple family of wellcharacterized exceptional graphs). For hamiltonicity, Chvátal's Conjecture [11] states that there exists a constant $t_{0}$ such that every $t_{0}$-tough graph on $n \geq 3$ vertices is hamiltonian, and it is proved in [2] that $t_{0} \geq 9 / 4$. For pancyclicity, the following theorem shows that there exists no such constant.

Theorem 1 (Brandt [7]) There are t-tough graphs with t arbitrarily large which are not weakly pancyclic.

Here a graph is called weakly pancyclic if it contains cycles of every length between the girth and the circumference. Hence, if a graph is not weakly pancyclic, then it is also not pancyclic. For more results about toughness and hamiltonian properties, we refer the interested reader to $[1,3,9]$. 
Forbidden subgraph conditions are an important type of sufficient conditions for the existence of Hamilton cycles and other hamiltonian properties of graphs. These structural conditions have a direct effect on the cycle and path properties of graphs. We refer to [8, 19-22] for more research about the structural implications and the cycle and path properties related to forbidden subgraph conditions. Over the years, researchers have established full characterizations of all possible single forbidden graphs and pairs of forbidden subgraphs ensuring that every 2-connected graph is hamiltonian. Some of these forbidden subgraph results give support for Bondy's metaconjecture, as shown by the following two theorems. We refer to Fig. 1 for some of the graphs that appear in the below statements.

Theorem 2 (Bedrossian [4]; Faudree and Gould [12]) Let $R$ and $S$ be connected graphs with $R, S \neq P_{3}$, and let $G$ be a 2-connected graph. Then $G$ being $\{R, S\}$ free implies $G$ is hamiltonian if and only if (up to symmetry) $R=K_{1,3}$ and $S=$ $P_{4}, P_{5}, P_{6}, C_{3}, Z_{1}, Z_{2}, B, N$ or $W$.

Theorem 3 (Bedrossian [4]) Let $R$ and $S$ be connected graphs with $R, S \neq P_{3}$, and let $G$ be a 2-connected graph which is not a cycle. Then $G$ being $\{R, S\}$-free implies $G$ is pancyclic if and only if (up to symmetry) $R=K_{1,3}$ and $S=P_{4}, P_{5}, Z_{1}$ or $Z_{2}$.

It can be observed that many of the nonhamiltonian graph families that show the necessity of forbidding certain subgraphs are not 1-tough. This fact caused researchers to consider using the necessary condition of being 1-tough instead of 2connected. In [18], Nikoghosyan posed several conjectures relating toughness and forbidden subgraph conditions to hamiltonicity. Motivated by one of these conjectures, $\mathrm{Li}$ et al. [16] considered single forbidden subgraphs under the condition of 1-toughness, and came up with the following results. Here, $G_{1} \cup G_{2}$ denotes the disjoint union of two vertex-disjoint graphs $G_{1}$ and $G_{2}$, and $k G$ denotes the disjoint union of $k$ copies of the graph $G$.

Theorem 4 (Li et al. [16]) Let $R$ be an induced subgraph of $P_{4}, K_{1} \cup P_{3}$ or $2 K_{1} \cup K_{2}$. Then every $R$-free 1-tough graph on at least three vertices is hamiltonian.

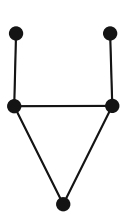

$B$

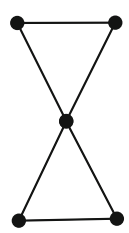

$H$

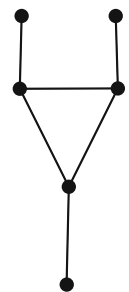

$N$

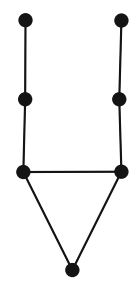

$D$

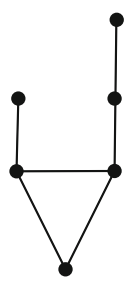

$W$

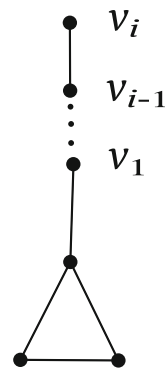

$Z_{i}$

Fig. 1 Graphs $B, H, N, D, W$ and $Z_{i}$ 
Theorem 5 ( $\mathrm{Li}$ et al. [16]) Let $R$ be a graph on at least three vertices. If every $R$ free 1-tough graph on at least three vertices is hamiltonian, then $R$ is an induced subgraph of $K_{1} \cup P_{4}$.

The question whether every 1-tough $K_{1} \cup P_{4}$-free graph is hamiltonian is still open, and seems very hard to answer. In fact, this was the conjecture of Nikoghosyan in [18] that motivated the work in [16]. Regarding hamiltonianconnectivity, one easily checks that every hamiltonian-connected graph has toughness strictly larger than one. In 1978, Jung [14] showed that for $P_{4}$-free graphs, the toughness condition $\tau(G)>1$ is a necessary and sufficient condition for hamiltonian-connectivity.

Theorem 6 (Jung [14]) Let $G$ be a $P_{4}$-free graph. Then $G$ is hamiltonian-connected if and only if $\tau(G)>1$.

Recently, this work was followed up by studying more cases of forbidden subgraphs for the property of hamiltonian-connectivity in [23]. By combining their results with Theorem 6, in [23] the authors concluded that the results on hamiltonian-connectivity are completely analogous to the hamiltonian case: every graph $H$ such that any 1-tough $H$-free graph is hamiltonian also ensures that every $H$-free graph with toughness larger than one is hamiltonian-connected. And similarly, there is no other forbidden subgraph having this property, except possibly for the graph $K_{1} \cup P_{4}$ itself.

Theorem 7 (Zheng et al. [23]) Let $R$ be an induced subgraph of $K_{1} \cup P_{3}$ or $2 K_{1} \cup K_{2}$. Then every $R$-free graph $G$ with $\tau(G)>1$ on at least three vertices is hamiltonian-connected.

Theorem 8 (Zheng et al. [23]) Let $R$ be a graph on at least three vertices. If every $R$-free graph $G$ with $\tau(G)>1$ on at least three vertices is hamiltonian-connected, then $R$ is an induced subgraph of $K_{1} \cup P_{4}$.

Turning to pancyclicity, inspired by Bondy's metaconjecture, we examined whether the condition in Theorem 4 in fact implies pancyclicity, and we obtained the following three results. We postpone the proofs of these results to Sects. 3, 4 and 5 , respectively.

Theorem 9 Let $G$ be a $K_{1} \cup P_{3}$-free 1-tough graph on $n \geq 3$ vertices. Then $G$ is pancyclic or $G \in\left\{C_{5}, K_{\frac{n}{2}, \frac{n}{2}}\right\}$.

Clearly, the latter case can only occur when $n$ is even. For the next result we first define the graph $C_{6}^{+}$and the class of graphs $\mathcal{K}^{-}$. The graph $C_{6}^{+}$is obtained from $C_{6}$ by adding an edge between two vertices at distance 2 in $C_{6}$. The class $\mathcal{K}^{-}$consists of all balanced bipartite graphs $K_{s, s}-M(s \geq 2)$, where $M$ is a matching of $K_{s, s}$ with $0 \leq|M| \leq s$.

Theorem 10 Let $G$ be a $2 K_{1} \cup K_{2}$-free 1-tough graph on at least three vertices. Then $G$ is pancyclic or $G \in \mathcal{K}^{-} \cup\left\{C_{5}, C_{6}^{+}\right\}$. 
Theorem 11 Let $G$ be a $P_{4}$-free 1-tough graph on $n \geq 3$ vertices. Then $G$ is pancyclic or $G=K_{\frac{n}{2}, \frac{n}{2}}$.

By Theorem 5, there is no graph $H$ other than the induced subgraphs of $K_{1} \cup P_{4}$ that can ensure every 1-tough $H$-free graph is hamiltonian. Hence, we obtain the following conclusion.

Theorem 12 Let $R$ be a graph on at least three vertices. If every $R$-free 1-tough graph $G$ on at least three vertices is pancyclic, then $R$ is an induced subgraph of $K_{1} \cup P_{4}$.

For the open case with $K_{1} \cup P_{4}$, it is natural to ask whether every $K_{1} \cup P_{4}$-free 1tough graph $G$ on at least three vertices is pancyclic (except possibly for some welldefined classes of graphs). Note that the exceptional graphs in the statements of Theorems 9-11 are also exceptions to $K_{1} \cup P_{4}$-free 1-tough graphs being pancyclic, and they are $C_{5}, C_{6}^{+}, K_{\frac{n}{2}, \frac{n}{2}}$ and $\mathcal{K}^{-}$. In fact, there are many other infinite classes of exceptional graphs for this statement. One of these classes is illustrated in Fig. 2. This class $\mathcal{C}_{5}^{s}$ consists of graphs that are obtained from a $C_{5}$ by replacing each vertex $v$ of the $C_{5}$ by an independent set $I_{v}$ of cardinality $s \geq 1$, and adding all edges between $I_{u}$ and $I_{v}$ whenever $u v$ is an edge of the $C_{5}$. These graphs clearly contain no $C_{3}$, so they are not pancyclic. They are hamiltonian (even if the sets $I_{v}$ have different cardinalities, as long as the graphs are 1-tough. We refer to [10], where these graphs are called $C_{5}^{*}$-type graphs and treated as special cases of triangle-free $2 K_{2}$-free graphs). Using that $C_{5}$ is $K_{1} \cup P_{4}$-free, it is easy to check that all these graphs are $K_{1} \cup P_{4}$-free. There are basically two choices for cut sets that should be considered for determining the toughness. One option is to delete two nonconsecutive sets $I_{v}$ and $I_{w}$, resulting in $s+1$ components; the other option is to delete an additional set $I_{z}$, resulting in $2 s$ components. The latter option determines the toughness if $s \geq 3$, i.e., the toughness of these graphs is $\frac{3}{2}$ if $s \geq 3$. Hence, the class of graphs $\mathcal{C}_{5}^{s}$ shows that even with a toughness strictly larger than one, there exist infinitely many exceptional graphs to the above statement. In a similar way, one can define the classes $\mathcal{C}_{4}^{s}$ (balanced complete bipartite graphs $K_{2 s, 2 s}$ ) and $\mathcal{C}_{6}^{s}$, based on a $C_{4}$ and $C_{6}$,

Fig. 2 Graphs $\mathcal{C}_{5}^{s}$

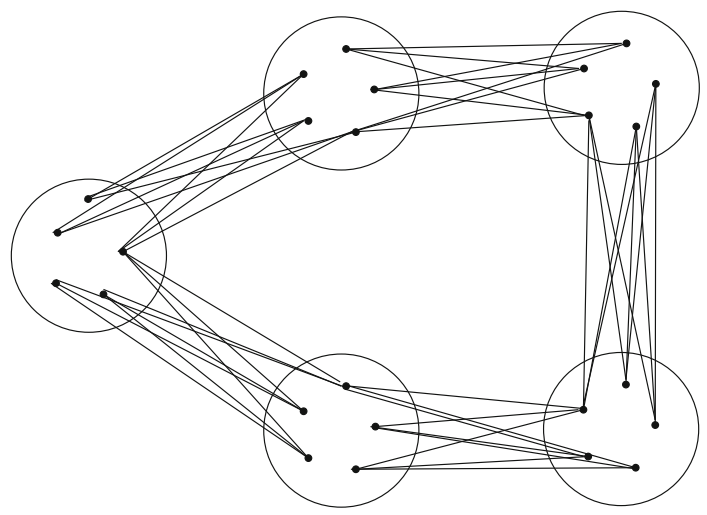


respectively. Graphs from these classes are also not pancyclic, $K_{1} \cup P_{4}$-free, and they have toughness equal to one. Here, we pose the following problem.

Problem 1 Except for $C_{6}^{+}$and the graphs from $K_{\frac{n}{2}, \frac{n}{2}}, \mathcal{K}^{-}, \mathcal{C}_{4}^{s}, \mathcal{C}_{5}^{s}$, and $\mathcal{C}_{6}^{s}$, are there any other 1-tough $K_{1} \cup P_{4}$-free graphs on at least three vertices that are not pancyclic?

In Theorems 9-11, all the exceptional graphs have toughness exactly one. Hence, recalling the above remarks on the class $\mathcal{C}_{5}^{s}$, we know that forbidding any proper induced subgraph of $K_{1} \cup P_{4}$ can ensure that a graph on at least three vertices with toughness larger than 1 is pancyclic, and $K_{1} \cup P_{4}$ itself does not have this property. In fact, we can prove that only the proper induced subgraphs of $K_{1} \cup P_{4}$ have this property.

Theorem 13 Let $R$ be a graph, and let $G$ be a graph on at least three vertices with $\tau(G)>1$. Then $G$ being $R$-free implies $G$ is pancyclic if and only if $R$ is a proper induced subgraph of $K_{1} \cup P_{4}$.

The remainder of the paper is devoted to the proofs of our main results, but we start with a short section containing some preliminaries.

\section{Preliminaries}

We call a cycle with $m$ vertices an $m$-cycle. Let $C$ be an $m$-cycle of $G$ with a given orientation, and denoted as $C=x_{1} x_{2} \ldots x_{m} x_{1}$. For a vertex $x_{i} \in V(C)(1 \leq i \leq m)$, let $x_{i}^{-l}, x_{i}^{+l}(1 \leq i-l<i+l \leq m)$ denote the vertices $x_{i-l}$ and $x_{i+l}$ on $C$, respectively. Instead of $x_{i}^{-1}$ and $x_{i}^{+1}$, we simply use $x_{i}^{-}$and $x_{i}^{+}$to denote the immediate predecessor and successor of $x_{i}$ on $C$, respectively. For two vertices $x_{i}, x_{j} \in V(C)$, $x_{i} C x_{j}$ denotes the subpath of $C$ from $x_{i}$ to $x_{j}$, and $x_{j} \bar{C} x_{i}$ denotes the path from $x_{j}$ to $x_{i}$ in the reverse direction. For any $I \subseteq V(C)$, let $I^{-}=\left\{x_{i}^{-} \mid x_{i} \in I\right\}$ and $I^{+}=\left\{x_{i}^{+} \mid x_{i} \in I\right\}$. A similar notation is used for paths. In the proofs, we often use $\{u, v, w, x\} \cong H$ as shorthand for $\{u, v, w, x\}$ induces a copy of $H$ in $G$.

The main idea of our proofs of Theorems 9-11 is as follows. First we consider a shortest cycle of the graph $G$. In case $G$ does not contain some specific short cycles, we characterize $G$ as one of the exceptional graphs. For the other case, we prove by contradiction that if $G$ contains a $k$-cycle, then it also contains a $(k+1)$-cycle for any integer $k \in\{3,4, \ldots, n-1\}$. By induction, this is sufficient to show that $G$ is pancyclic. All our proofs are modelled along these lines and look similar, but contain different argumentations. In particular, our proofs considerably differ in length. 


\section{Proof of Theorem 9}

Suppose that $G$ is a graph satisfying the conditions of Theorem 9. Since $G$ is 1tough, $G$ contains a cycle. Clearly, the shortest (induced) cycle of $G$ is $C_{3}, C_{4}$ or $C_{5}$; otherwise, $G$ has an induced subgraph isomorphic to $K_{1} \cup P_{3}$. We first prove the following claim to characterize the exceptional graphs.

Claim 1 If the shortest cycle of $G$ is $C_{5}$, then $G$ is $C_{5}$; if the shortest cycle of $G$ is $C_{4}$, then $G$ is $K_{s, s}(s \geq 2)$.

Proof Suppose that the shortest cycle of $G$ is $C_{5}$. Let $C=v_{1} v_{2} \ldots v_{5} v_{1}$ be a shortest cycle. Then $C$ is an induced cycle. If $|V(G)|=5$, then $G=C_{5}$, and the claim holds. Now we assume that $|V(G)| \geq 6$ and $x$ is a vertex of $G-V(C)$. To avoid $\left\{x, v_{1}, v_{2}, v_{3}\right\}$ inducing a $K_{1} \cup P_{3}$, we have that $x$ is adjacent to $v_{1}, v_{2}$ or $v_{3}$. Without loss of generality, we assume $x v_{1} \in E(G)$. If $N(x) \cap\left\{v_{2}, v_{3}, v_{4}\right\} \neq \emptyset$, then $G$ has a $C_{3}$ or $C_{4}$, contradicting the fact that $C_{5}$ is a shortest cycle. If $N(x) \cap\left\{v_{2}, v_{3}, v_{4}\right\}=\emptyset$, then $\left\{x, v_{2}, v_{3}, v_{4}\right\}$ induces a copy of $K_{1} \cup P_{3}$, a contradiction. Hence, $G$ has 5 vertices and $G$ is $C_{5}$.

Suppose that the shortest cycle of $G$ is $C_{4}$. Let $C=v_{1} v_{2} \ldots v_{4} v_{1}$ be a shortest cycle. Hence $C$ is an induced cycle. If $G$ has 4 vertices, then $G=C_{4}=K_{2,2}$, and the claim holds. If $G$ is not $C_{4}$, then there is a vertex $x_{0}$ in $G-V(C)$. Similar to the above case, without loss of generality, we assume $x_{0} v_{1} \in E(G)$. To avoid inducing $K_{1} \cup P_{3}$ and $C_{3}$, we have that $N\left(x_{0}\right) \cap\left\{v_{2}, v_{3}, v_{4}\right\}=\left\{v_{3}\right\}$. Using the same arguments, we have that every vertex $x_{i}$ of $G-V(C)$ has two neighbors on $C$, which are $\left\{v_{1}, v_{3}\right\}$ or $\left\{v_{2}, v_{4}\right\}$. For two vertices $x_{i}, x_{i} \in G-V(C)$, if $x_{i}$ and $x_{j}$ have the same neighbors on $C$, then $x_{i} x_{j} \notin E(G)$; otherwise $x_{i}, x_{j}$ and one of their neighbors on $C$ induce a copy of $C_{3}$, a contradiction. If $x_{i}$ and $x_{j}$ have different neighbors on $C$, without loss of generality assume that $N_{C}\left(x_{i}\right)=\left\{v_{1}, v_{3}\right\}$ and $N_{C}\left(x_{j}\right)=\left\{v_{2}, v_{4}\right\}$. Then $x_{i} x_{j} \in E(G)$; otherwise $\left\{x_{i}, x_{j}, v_{2}, v_{4}\right\} \cong K_{1} \cup P_{3}$, a contradiction. Now denote $A=N_{G}\left(v_{1}, v_{3}\right), B=N_{G}\left(v_{2}, v_{4}\right)$. Then $V(G)=A \cup B$. Moreover, $A, B$ are two independent vertex sets and every vertex of $A$ is adjacent to every vertex of $B$. Hence, $G$ is a complete bipartite graph, and according to the toughness, $G$ is a balanced complete bipartite graph $K_{s, s}$.

By Claim 1, we see that if $G$ has no $C_{3}$, then $G$ is either $C_{5}$ or $K_{s, s}$. Next, we suppose that $G$ is neither $C_{5}$ nor $K_{s, s}$. This implies that $G$ contains a $C_{3}$. We show that $G$ is pancyclic by proving the following fact.

Fact If $G$ has a $k$-cycle $(k=3,4, \ldots, n-1)$, then $G$ has a $(k+1)$-cycle.

Proof Suppose, by contradiction, that $G$ has a $k$-cycle, but no $(k+1)$-cycle, for some $k \in\{3,4, \ldots, k-1\}$. Let $C=v_{1} v_{2} \ldots v_{k} v_{1}$ be a $k$-cycle, and let $H$ be a component of $G-V(C)$. Since $G$ is 1-tough, $H$ has at least two neighbors on $C$. We distinguish two cases.

Case 1. $H$ has two neighbors that are consecutive on $C$.

Clearly, $H$ is not trivial in this case. We prove a number of claims before we complete the proof for this case. 
Claim $2 H$ contains an edge ab such that $a v_{i} \in E(G)$ and $b v_{i+1} \in E(G)$, $i \in\{1,2, \ldots, k\}$.

Proof Choose two consecutive neighbors of $H$ on $C$ such that their distance in $H$ is shortest. Without loss of generality, assume that $v_{1}, v_{2}$ are such vertices, and $a, b \in$ $V(H)$ are neighbors of $v_{1}$ and $v_{2}$, respectively. We have that $a \neq b$; otherwise $G$ has a $(k+1)$-cycle $v_{1} a v_{2} C v_{1}$, contradicting the assumption that $G$ has no $(k+1)$-cycle. Let $P=a x_{1} x_{2} \ldots x_{s} b$ be a shortest $(a, b)$-path in $H$. Clearly, $V(P) \cap N\left(v_{1}\right)=\{a\}$ and $V(P) \cap N\left(v_{2}\right)=\{b\}$. Then we have $s \leq 1$; otherwise $\left\{v_{2}, a, x_{1}, x_{2}\right\} \cong K_{1} \cup P_{3}$, a contradiction. Suppose that $s=1$ and $P=a x_{1} b$. By the choice of $v_{1}, v_{2}$ and the assumption that $G$ has no $(k+1)$-cycle, we have that $\left\{x_{1}, b\right\} \cap N\left(v_{3}\right)=\emptyset$ and $\left\{a, x_{1}\right\} \cap N\left(v_{k}\right)=\emptyset$. If $k=3$, i.e., $v_{k}=v_{3}$, then $\left\{v_{k}, a, x_{1}, b\right\} \cong K_{1} \cup P_{3}$, a contradiction. Assume that $k \geq 4$. To avoid $\left\{v_{k}, a, x_{1}, b\right\}$ and $\left\{v_{3}, a, x_{1}, b\right\}$ inducing $K_{1} \cup P_{3}$, we have $b v_{k} \in E(G)$ and $a v_{3} \in E(G)$. To avoid $\left\{v_{3}, x_{1}, b, v_{k}\right\}$ inducing $K_{1} \cup P_{3}$, we have $v_{3} v_{k} \in E(G)$. If $k=4$, then $v_{1} a x_{1} b v_{2} v_{1}$ is a $(k+1)$-cycle, a contradiction. If $k \geq 5$, then according to the choice of $v_{1}, v_{2}$ and the assumption that $G$ has no $(k+1)$-cycle we have that $v_{4} a, v_{4} x_{1} \notin E(G)$. To avoid $\left\{v_{4}, a, x_{1}, b\right\}$ inducing $K_{1} \cup P_{3}$, we have $v_{4} b \in E(G)$, but then $v_{3} a x_{1} b v_{4} C v_{k} v_{3}$ is a $(k+1)$-cycle, a contradiction. Hence, $s=0$ and the claim holds.

By Claim 2 and the assumptions, we have that $k \geq 4$. Without loss of generality, assume that $a b \in E(H)$ and $a v_{1} \in E(G), b v_{2} \in E(G)$. We next prove the following claim.

Claim $3 a v_{3} \in E(G)$.

Proof Suppose that $a v_{3} \notin E(G)$. To avoid $\left\{v_{3}, b, a, v_{1}\right\}$ inducing $K_{1} \cup P_{3}$, we have $v_{1} v_{3} \in E(G)$. We also have that $a v_{4} \notin E(G)$ and $b v_{4} \notin E(G)$; otherwise, $v_{2} b a v_{4} C v_{2}$ or $v_{2} b v_{4} C v_{1} v_{3} v_{2}$ is a $(k+1)$-cycle, respectively. To avoid $\left\{v_{4}, a, b, v_{2}\right\}$ inducing $K_{1} \cup P_{3}$, we have $v_{2} v_{4} \in E(G)$. Then $v_{1} a b v_{2} v_{4} C v_{1}$ is a $(k+1)$-cycle, a contradiction.

We now divide $V(C)$ into two sets. Let $A=\left\{v_{i} \mid i\right.$ is odd, $\left.1 \leq i \leq k\right\}$, and $B=$ $\left\{v_{i} \mid i\right.$ is even, $\left.1 \leq i \leq k\right\}$. Then clearly $V(C)=A \cup B$. We prove the following claim on the structure of $A$ and $B$.

Claim $4 \quad k$ is even, and $A \subseteq N(a) \backslash N(b), B \subseteq N(b) \backslash N(a)$. Moreover, $A$ and $B$ are independent sets, and each vertex of $A$ is adjacent to each vertex of $B$.

Proof We use induction to prove that $A \subseteq N(a) \backslash N(b)$ and $B \subseteq N(b) \backslash N(a)$. First, we show that $v_{4} \in N(b) \backslash N(a)$. Since $a v_{3} \in E(G), a v_{4} \notin E(G)$. We also have $v_{2} v_{4} \notin E(G)$; otherwise, $v_{1} a b v_{2} v_{4} C v_{1}$ is a $(k+1)$-cycle, a contradiction. Hence $v_{4} \in N(b)$; otherwise $\left\{v_{4}, a, b, v_{2}\right\} \cong K_{1} \cup P_{3}$, a contradiction. Next, we show that if $v_{s-1} \in N(a), v_{s} \in N(b) \quad(s \geq 4)$, then $v_{s+1} \in N(a) \backslash N(b)$. First, $v_{s+1} \notin N(b)$ since $v_{s} \in N(b)$. We have $v_{s-1} v_{s+1} \notin E(G)$; otherwise, $v_{1} a b v_{2} C v_{s-1} v_{s+1} C v_{1}$ is a $(k+1)$ cycle, a contradiction. To avoid $\left\{v_{s+1}, b, a, v_{s-1}\right\}$ inducing a $K_{1} \cup P_{3}$, we have $v_{s+1} \in N(a)$. By a similar analysis, we get that if $v_{s-1} \in N(b), v_{s} \in N(a)(s \geq 4)$, then $v_{s+1} \in N(b) \backslash N(a)$. Thus, $V(C) \subseteq N(a) \cup N(b)$, and $N_{C}(a)$ and $N_{C}(b)$ occur alternately on $C$. Since $v_{1} \in N(a), v_{k} \in N(b)$. Hence, $k$ is even, and $A \subseteq N(a) \backslash N(b)$, 
$B \subseteq N(b) \backslash N(a)$.

Suppose that $v_{i}, v_{j} \in A$ and $v_{i} v_{j} \in E(G)$. Then $v_{i+1}, v_{j+1} \in B \subseteq N(b)$, and there is a $(k+1)$-cycle $v_{i+1} b v_{j+1} C v_{i} v_{j} \bar{C} v_{i+1}$, a contradiction. Hence, $A$ is an independent set. Similarly, $B$ is also an independent set. Suppose that there is a pair of vertices $v_{i} \in A, v_{j} \in B$ such that $v_{i} v_{j} \notin E(G)$. We have that $v_{j} \neq v_{i+1}$, and since $\left\{v_{j}, v_{i+1}\right\} \in B$, we have $v_{j} v_{i+1} \notin E(G)$. Then $\left\{v_{j}, a, v_{i}, v_{i+1}\right\} \cong K_{1} \cup P_{3}$, a contradiction. Hence, $v_{i} v_{j} \in E(G)$ for any $v_{i} \in A, v_{j} \in B$, and the claim holds.

We need two more claims before we can complete our proof for this case.

Claim $5 G-V(C)=H$.

Proof Suppose, by contradiction, that $H^{\prime}$ is another component distinct from $H$ of $G-V(C)$. Then there is a vertex $y \in V\left(H^{\prime}\right)$ such that $y$ has a neighbor on $C$. By symmetry, assume $y v_{1} \in E(G)$. If $y$ has another neighbor $v_{i}$ on $C$ distinct from $v_{1}$, then $v_{i} \notin A$; otherwise, $\left\{b, v_{1}, y, v_{i}\right\} \cong K_{1} \cup P_{3}$, a contradiction. Thus, $v_{i} \in$ $B \backslash\left\{v_{2}, v_{k}\right\}$ and $k \neq 4$. By Claim $4, v_{i+1} v_{2} \in E(G)$. Then $v_{i} y v_{1} \bar{C} v_{i+1} v_{2} C v_{i}$ is a $(k+1)$-cycle, a contradiction. Hence $y$ has only one neighbor on $C$. Since $G$ is 1 tough, there is another vertex $y^{\prime}$ in $V\left(H^{\prime}\right)$, and $y^{\prime}$ has a neighbor on $C$ distinct from $v_{1}$. By the same arguments, $y^{\prime}$ has only one neighbor on $C$, say $v_{i}$. If $y y^{\prime} \in E(G)$, then $\left\{b, y^{\prime}, y, v_{1}\right\} \cong K_{1} \cup P_{3}$, a contradiction. If $y y^{\prime} \notin E(G)$, then there is an induced $P_{3}$ in $H^{\prime}$, and this induced $P_{3}$ together with the vertex $a$ in $H$ will induce a $K_{1} \cup P_{3}$, a contradiction. Hence, $G-V(C)=H$.

By Claim 5, we know that $H$ is the only component of $G-V(C)$, and $a b$ is an edge of $H$. We denote $A^{\prime}=N_{H}(a)$ and $B^{\prime}=N_{H}(b)$.

Claim 6 The following properties hold:

(1) $V(H)=A^{\prime} \cup B^{\prime}$ and $A^{\prime} \cap B^{\prime}=\emptyset$.

(2) each vertex of $A^{\prime}$ is adjacent to each vertex of $B$, and each vertex of $B^{\prime}$ is adjacent to each vertex of $A$.

(3) $A \cup A^{\prime}$ and $B \cup B^{\prime}$ are independent sets.

(4) each vertex of $A^{\prime}$ is adjacent to each vertex of $B^{\prime}$.

Proof We prove the properties in the same order.

(1) If $V(H)=\{a, b\}$, then the claim holds. Now we suppose that $V(H) \neq\{a, b\}$ and by contradiction, we suppose that $V(H) \neq A^{\prime} \cup B^{\prime}$. There is a vertex $x \in$ $V(H) \backslash\left(A^{\prime} \cup B^{\prime}\right)$ such that $x a_{1} \in E(G)$ or $x b_{1} \in E(G)$, where $a_{1} \in A^{\prime}$ and $b_{1} \in B^{\prime}$. Without loss of generality, assume that $x a_{1} \in E(G)$. If $x$ is adjacent to a vertex $v_{i}$ of $B$, then by Claim 4 we have that $v_{i} v_{i+3} \in E(G)$, and there is a $(k+1)$-cycle $v_{i-1} a a_{1} x v_{i} v_{i+3} C v_{i-1}$ (if $\left.k=4, v_{i+3}=v_{i-1}\right)$, a contradiction. Thus, $x$ has no neighbor in $B$. For a vertex $v_{i}$ of $B,\left\{x, a, b, v_{i}\right\}$ induces a $K_{1} \cup P_{3}$, a contradiction. Hence, $V(H)=A^{\prime} \cup B^{\prime}$. Suppose that $x^{\prime} \in A^{\prime} \cap B^{\prime}$. Then there is a $(k+1)$-cycle $v_{1} a x^{\prime} b v_{2} v_{5} C v_{1}$ (possible $\left.v_{5}=v_{1}\right)$, a contradiction. Hence, $A^{\prime} \cap B^{\prime}=\emptyset$. 
(2) Let $a_{1}$ be a vertex of $A^{\prime}$. From (1), we have $b a_{1} \notin E(G)$. Since $k \geq 4$, $|A|=|B| \geq 2$. If there are two vertices $v_{i}, v_{j} \in B$ such that $a_{1} v_{i} \notin E(G)$ and $a_{1} v_{j} \notin E(G)$, then $\left\{a_{1}, v_{i}, b, v_{j}\right\} \cong K_{1} \cup P_{3}$, a contradiction. Thus, there is at most one vertex of $B$ that is not adjacent to $a_{1}$. Suppose that $a_{1} v_{i} \notin E(G)$ and $a_{1} v_{j} \in E(G)\left(v_{i}, v_{j} \in B\right)$. Then $\left\{v_{i}, a, a_{1}, v_{j}\right\} \cong K_{1} \cup P_{3}$, a contradiction. Hence, $a_{1}$ is adjacent to each vertex of $B$. By the arbitrary selection of $a_{1}$, each vertex of $A^{\prime}$ is adjacent to each vertex of $B$. By symmetry, each vertex of $B^{\prime}$ is adjacent to all the vertices of $A$.

(3) First, $A^{\prime}$ is independent set; otherwise, suppose $a_{1}, a_{2} \in A^{\prime}$ and $a_{1} a_{2} \in E(G)$. Then by (2), there is a $(k+1)$-cycle $v_{1} a a_{1} a_{2} v_{4} C v_{1}$, a contradiction. Similarly, $B^{\prime}$ is also an independent set. Next, $N\left(A^{\prime}\right) \cap A=\emptyset$; otherwise, suppose that $a_{1} \in A^{\prime}$ and $v_{i} \in A$ such that $a_{1} v_{i} \in E(G)$. Then there is a $(k+1)$-cycle $v_{i-2} a a_{1} v_{i} C v_{i-2}$, a contradiction. Hence, $A \cup A^{\prime}$ is an independent set. Similarly, $B \cup B^{\prime}$ is also an independent set.

(4) Suppose that $a_{1} \in A^{\prime}, b_{1} \in B^{\prime}$ and $a_{1} b_{1} \notin E(G)$. By (2) and (3), for any pair of vertices $v_{i}, v_{j} \in A,\left\{a_{1}, v_{i}, b_{1}, v_{j}\right\} \cong K_{1} \cup P_{3}$, a contradiction. Hence, each vertex of $A^{\prime}$ is adjacent to each vertex of $B^{\prime}$.

From Claims 4-6, we have that $G$ is a complete bipartite graph with two independent sets $A \cup A^{\prime}$ and $B \cup B^{\prime}$. Since $G$ is 1-tough, $G$ is a balanced complete bipartite graph $K_{s, s}$, contradicting the assumption. This completes the proof for Case 1.

Case 2. For every component $H$ of $G-V(C)$, any two neighbors of $H$ on $C$ are not consecutive.

Let $N_{C}(H)=\left\{u_{1}, u_{2}, \ldots, u_{s}\right\} \quad\left(s \geq 2\right.$, and with all $u_{i}$ chosen in this order according to the orientation of $C$ ). The $s$ neighbors of $H$ on $C$ divide the cycle $C$ into $s$ segments, denoted by $S_{i}=u_{i}^{+} C u_{i+1}\left(i=1,2, \ldots, s\right.$, and with $\left.u_{s+1}=u_{1}\right)$, so with $\left|S_{i}\right| \geq 2$ for any $i \in\{1,2, \ldots, s\}$. Since $G$ is 1-tough, there are at least two segments that are connected by a path internally-disjoint with $V(C) \cup V(H)$. As we will see, the choice of these two segments is irrelevant for the remainder of the proof. So we ignore the indices, and assume without loss of generality that $S_{1}$ and $S_{2}$ are connected by such a path. We choose a path $P$ with two end vertices $y \in S_{1}, y^{\prime} \in S_{2}$ such that the path $u_{1}^{+} C y P y^{\prime} \bar{C} u_{2}^{+}$is as short as possible. Then we have that $|V(P)|=$ 2 and $y=u_{1}^{+}, y^{\prime}=u_{2}^{+}$; otherwise, the path $u_{1}^{+} C y P y^{\prime} \bar{C} u_{2}^{+}$contains an induced $P_{3}$. Combining that induced $P_{3}$ with one vertex of $H$ we get an induced $K_{1} \cup P_{3}$, a contradiction. Thus, $u_{1}^{+} u_{2}^{+} \in E(G)$. Suppose that $a u_{1} \in E(G), b u_{2} \in E(G)$ for $a, b \in V(H)$. We have $a \neq b$; otherwise, $u_{1} a u_{2} \bar{C} u_{1}^{+} u_{2}^{+} C u_{1}$ is a $(k+1)$-cycle. We also have that $a b \in E(G)$; otherwise, $H$ contains an induced $P_{3}$, and combining that induced $P_{3}$ with $u_{1}^{+}$we get an induced $K_{1} \cup P_{3}$, a contradiction. If $u_{1}^{+}=u_{2}^{-}$, then $u_{1} a b u_{2} C u_{1}$ is a $(k+1)$-cycle, a contradiction. Hence, $u_{1}^{+} \neq u_{2}^{-}$. If $u_{1}^{++} u_{2}^{+} \in E(G)$, then $u_{1} a b u_{2} \bar{C} u_{1}^{++} u_{2}^{+} C u_{1}$ is a $(k+1)$-cycle, a contradiction. Hence, $u_{1}^{++} u_{2}^{+} \notin E(G)$. Then $\left\{a, u_{1}^{++}, u_{1}^{+}, u_{2}^{+}\right\} \cong K_{1} \cup P_{3}$, our final contradiction.

This completes the proof of Theorem 9 . 


\section{Proof of Theorem 10}

Suppose that $G$ is a graph satisfying the conditions of Theorem 10 . Since $G$ is 1tough, $G$ contains a cycle. The shortest (induced) cycle of $G$ is $C_{3}, C_{4}, C_{5}$ or $C_{6}$; otherwise, $G$ clearly has an induced subgraph isomorphic to $2 K_{1} \cup K_{2}$. We again start by proving a number of claims.

Claim 1 If the shortest cycle of $G$ is $C_{5}$ or $C_{6}$, then $G$ is $C_{5}$ or $C_{6}$, respectively.

Proof Suppose that the shortest cycle of $G$ is $C_{5}$. Let $C=v_{1} v_{2} \ldots v_{5} v_{1}$ be an induced 5-cycle. If $G$ is not $C_{5}$, then there is a vertex $u \in G-V(C)$ such that $u$ is adjacent to a vertex of $C$, say $v_{1}$. Since $G$ has neither a $C_{3}$ nor a $C_{4}, u$ has no other neighbor on $C$ distinct from $v_{1}$. Now $\left\{u, v_{2}, v_{4}, v_{5}\right\} \cong 2 K_{1} \cup K_{2}$, a contradiction. In the same way, we can prove that if the shortest cycle of $G$ is $C_{6}$, then $G$ is $C_{6}$.

Claim 2 If the shortest cycle of $G$ is $C_{4}$, then $G$ is $C_{4}$ or $K_{s, s}-M$, where $s \geq 3$ and $M$ is a matching of $K_{s, s}$ with $0 \leq|M| \leq s$.

Proof Suppose that $w$ is a vertex of $G$ with the maximum degree. If $d(w)=2$, then $G$ is $C_{4}$, and the claim holds. Assume that $d(w) \geq 3$. Now we draw the graph $G$ arranged as a rooted tree: $w$ is the root and denoted as the first layer $L_{1}$, all the neighbors of $w$ are arranged as the second layer $L_{2}$, all the new neighbors of vertices of $L_{2}$ (where new means that the vertices do not appear in the existing layers) are arranged as the third layer $L_{3}$, etc., until all layers together cover $V(G)$. By this labeling method, we know that there is no edge between $L_{i}$ and $L_{j}$ if $i \neq j-1$ and $i \neq j+1$. Since $G$ has no $C_{3}, L_{2}$ is an independent set. Next we prove three subclaims on the structure of the layers.

Claim 2.1 G has at most 4 layers.

Proof Suppose that $G$ has 5 or more layers. Let $u_{1}, u_{2}$ be two vertices of $L_{2}$, and let $x$ and $y$ be two vertices of $L_{4}$ and $L_{5}$, respectively, such that $x y \in E(G)$. Then $\left\{u_{1}, u_{2}, x, y\right\} \cong 2 K_{1} \cup K_{2}$, a contradiction.

Let $l_{i}=\left|L_{i}\right|$ for $i=1,2,3,4$. Then we denote $L_{1}=\{w\}, L_{2}=\left\{u_{1}, u_{2}, \ldots, u_{l_{2}}\right\}$, $L_{3}=\left\{v_{1}, v_{2}, \ldots, v_{l_{3}}\right\}, L_{4}=\left\{z_{1}, z_{2}, \ldots, z_{l_{4}}\right\}$.

Claim 2.2 $L_{4}=\left\{z_{1}\right\}$ or $L_{4}=\emptyset$.

Proof If $L_{4}$ has 2 or more vertices, then $\left\{z_{1}, z_{2}, w, u_{1}\right\} \cong 2 K_{1} \cup K_{2}$ (if $z_{1} z_{2} \notin E(G)$ ) or $\left\{u_{1}, u_{2}, z_{1}, z_{2}\right\} \cong 2 K_{1} \cup K_{2}$ (if $z_{1} z_{2} \in E(G)$ ), a contradiction.

Claim 2.3 $L_{3}$ is independent set.

Proof By contradiction, suppose that $v_{i} v_{j}$ is an edge of $L_{3}$. Since $G$ has no $C_{3}, v_{i}$ and $v_{j}$ have no common neighbor in $L_{2}$. Assume that $u_{i} v_{i}, u_{j} v_{j} \in E(G)$ and $u_{i} \neq u_{j}$. We have that $u_{i}, u_{j}$ have no common neighbor in $L_{3}$. Otherwise, suppose that $v_{k}$ is a common neighbor of $u_{i}$ and $u_{j}$ in $L_{3}$. Obviously, $v_{i} v_{k}, v_{j} v_{k} \notin E(G)$; otherwise $G$ contains a $C_{3}$. Then $\left\{w, v_{k}, v_{i}, v_{j}\right\} \cong 2 K_{1} \cup K_{2}$, a contradiction. Moreover, $N_{L_{3}}\left(u_{i}\right)=$ $\left\{v_{i}\right\}$ and $N_{L_{3}}\left(u_{j}\right)=\left\{v_{j}\right\}$. Otherwise, suppose that $v_{k} \in N_{L_{3}}\left(u_{i}\right) \backslash\left\{v_{i}\right\}$. Clearly, 
$v_{i} v_{k} \notin E(G)$; otherwise $G$ contains a $C_{3}$. Then $\left\{v_{i}, v_{k}, w, u_{j}\right\} \cong 2 K_{1} \cup K_{2}$, a contradiction. For any vertex $u_{k} \in L_{2} \backslash\left\{u_{i}, u_{j}\right\}$, we have that $N_{L_{3}}\left(u_{k}\right)=\left\{v_{i}\right\}$ or $N_{L_{3}}\left(u_{k}\right)=\left\{v_{j}\right\}$. Otherwise, suppose that $v_{k}$ is a vertex of $L_{3}$ different from $v_{i}, v_{j}$ that is adjacent to $u_{k}$. Then $\left\{u_{i}, u_{j}, u_{k}, v_{k}\right\} \cong 2 K_{1} \cup K_{2}$, a contradiction. Thus, $L_{3}=$ $\left\{v_{1}, v_{2}\right\}$ and every vertex of $L_{2}$ has degree 2 . Since $G$ is 1 -tough and $L_{2}$ is independent, $l_{2} \leq 3$. Since $l_{2}=d(w) \geq 3, l_{2}=3$. Without loss of generality, assume that $u_{1} v_{1}, u_{2} v_{2}, u_{3} v_{2} \in E(G)$. Then $\left\{u_{2}, u_{3}, u_{1}, v_{1}\right\} \cong 2 K_{1} \cup K_{2}$, a contradiction.

To complete the proof of Claim 2, we consider two cases.

Case A. $L_{4}=\left\{z_{1}\right\}$. Suppose that $v_{1}$ is a neighbor of $z_{1}$ in $L_{3}$. Since $G$ is 1-tough, the two independent sets $L_{2}$ and $L_{3}$ obviously have the same order, i.e., $l_{2}=l_{3} \geq 3$. If there is a vertex $v_{i} \in L_{3} \backslash\left\{v_{1}\right\}$ such that $v_{i} z_{1} \notin E(G)$, then $\left\{w, v_{i}, v_{1}, z_{1}\right\} \cong 2 K_{1} \cup K_{2}$, a contradiction. Hence, $N\left(z_{1}\right)=L_{3}$. If there are two vertices $u_{i}, u_{j} \in L_{2}$ that are not adjacent to a vertex $v_{k} \in L_{3}$, then $\left\{u_{i}, u_{j}, v_{k}, z_{1}\right\} \cong 2 K_{1} \cup K_{2}$, a contradiction. If all the vertices of $L_{2}$ are neighbors of $v_{k}$, then $d\left(v_{k}\right)=l_{2}+1>d(w)$, contradicting the assumption that $w$ is a vertex with maximum degree. Thus, every vertex of $L_{3}$ has exactly $l_{2}-1$ neighbors in $L_{2}$. Similarly, every vertex of $L_{2}$ has exactly $l_{3}-1$ neighbors in $L_{3}$. Hence, $G$ is a balanced bipartite graph $K_{s, s}-M^{*}$ with two independent vertex sets $L_{2} \cup\left\{z_{1}\right\}$ and $L_{3} \cup\{w\}$, where $s=l_{2}+1=l_{3}+1$ and $M^{*}$ is a perfect matching of $K_{s, s}$.

Case B. $L_{4}=\emptyset$. Since $G$ is 1 -tough, $l_{2}=l_{3}+1$. For a vertex $u_{k} \in L_{2}$, if there are two vertices $v_{i}, v_{j} \in L_{3}$ that are not neighbors of $u_{k}$, then $\left\{v_{i}, v_{j}, u_{k}, w\right\} \cong 2 K_{1} \cup K_{2}$, a contradiction. Thus, every vertex of $L_{2}$ has at least $l_{3}-1$ neighbors in $L_{3}$, and has degree at least $l_{3}$ in the graph $G$. For a vertex $v_{k} \in L_{3}$, suppose that $v_{k} u_{k} \in E(G)$. If there are two vertices $u_{i}, u_{j} \in L_{2}$ that are not neighbors of $v_{k}$, then $\left\{u_{i}, u_{j}, v_{k}, u_{k}\right\} \cong 2 K_{1} \cup K_{2}$, a contradiction. Thus, every vertex of $L_{3}$ has at least $l_{2}-1=l_{3}$ neighbors in $L_{3}$. Hence, $G$ is a balanced bipartite graph $K_{s, s}-M$ with two independent vertex sets $L_{2}$ and $\{w\} \cup L_{3}$, where $s=l_{2}=l_{3}+1$ and $M$ is a matching of $K_{s, s}$ with $0 \leq|M| \leq s-1$.

In both cases, we conclude that $G$ is either $C_{4}$ or $K_{s, s}-M$, where $s \geq 3$ and $M$ is a matching of $K_{s, s}$ with $0 \leq|M| \leq s$. This completes the proof of Claim 2.

Claim 3 If the minimum cycle of $G$ is $C_{3}$ and $G \neq C_{3}$, then $G$ contains $C_{4}$, unless $G=G_{1}$, where $G_{1}$ is the graph obtained by adding an edge to $C_{6}$ between two vertices at distance 2 in $C_{6}$.

Proof Suppose that $C=a b c a$ is a 3-cycle of $G$. For a component $H$ of $G-V(C)$, $H$ has at least two neighbors on $C$. If one vertex of $H$ has two neighbors on $C$, then $G$ contains $C_{4}$, and the claim holds. Assume that each vertex of $H$ is adjacent to at most one vertex of $C$. Suppose that $a_{1}, b_{1} \in V(H)$ and $a a_{1}, b b_{1} \in E(G)$. If $a_{1} b_{1} \in E(G)$, then $G$ contains $C_{4}$, and the claim holds. Assume that $a_{1} b_{1} \notin E(G)$. Let $P=a_{1} x_{1} x_{2} \ldots x_{s} b_{1}$ be a shortest $\left(a_{1}, b_{1}\right)$-path in $H$. We prove the following claims.

Claim $3.1 s=1$, i.e., $P=a_{1} x_{1} b_{1}$. 
Proof Suppose that $s \geq 2$. Since $P$ is a shortest path, $x_{1} b_{1} \notin E(G)$. If $x_{1} c \in E(G)$, then $G$ contains $C_{4}$, and the claim holds. Assume $x_{1} c \notin E(G)$. Then $\left\{b_{1}, c, a_{1}, x_{1}\right\} \cong 2 K_{1} \cup K_{2}$, a contradiction.

Claim 3.2 $H=P$.

Proof Suppose, by contradiction, that $w \in V(H) \backslash\left\{a_{1}, x_{1}, b_{1}\right\}$. If $w a_{1} \in E(G)$ and $w b_{1} \in E(G)$, then $a_{1} w b_{1} x_{1} a_{1}$ is a 4-cycle, and the claim holds. Without loss of generality, we assume that $w b_{1} \notin E(G)$. If $w a_{1} \in E(G)$ and $w c \in E(G)$, then $a_{1} w c a a_{1}$ is a 4-cycle, and the claim holds. If $w a_{1} \in E(G)$ and $w c \notin E(G)$, then $\left\{c, b_{1}, a_{1}, w\right\} \cong 2 K_{1} \cup K_{2}, \quad$ a contradiction. Thus, $w a_{1} \notin E(G), \quad$ and $N_{H}(a) \cup N_{H}(b)=\left\{x_{1}\right\}$. There must be a vertex $w^{\prime} \in V(H) \backslash\left\{a_{1}, x_{1}, b_{1}\right\}$ such that $w^{\prime} x_{1} \in E(G)$ (possibly $w^{\prime}=w$ ). If $w^{\prime} b \in E(G)$, then $w^{\prime} b b_{1} x_{1} w^{\prime}$ is a 4-cycle, and the claim holds. Assume that $w^{\prime} b \notin E(G)$. If $w^{\prime} c \in E(G)$, then $\left\{a_{1}, b_{1}, w^{\prime}, c\right\} \cong 2 K_{1} \cup K_{2}$, a contradiction. If $w^{\prime} c \notin E(G)$, then $\left\{a_{1}, w^{\prime}, b, c\right\} \cong$ $2 K_{1} \cup K_{2}$, a contradiction. Hence, $H=P$.

By Claim 3.2, $H=a_{1} x_{1} b_{1}$. If $x_{1}$ is adjacent to a vertex of $C$, then $G$ contains $C_{4}$, and the claim holds. Assume that $x_{1}$ has no neighbor on $C$. If $G-V(C)$ has no other component than $H$, then $G$ is $G_{1}$, and the claim holds. If $H^{\prime}$ is a component of $G-V(C)$ different from $H$, then $H^{\prime}$ is trivial; otherwise, an edge of $H^{\prime}$ with $a_{1}, b_{1}$ will induce a $2 K_{1} \cup K_{2}$, a contradiction. Since $G$ is 1-tough, the vertex of $H^{\prime}$ has two neighbors on $C$. Thus, $G$ contains $C_{4}$, and the claim holds.

By Claims 1 and 2, if $G$ has no 3-cycle, then $G$ is $C_{4}, C_{5}, C_{6}$ or $K_{s, s}-M$, where $s \geq 3$ and $M$ is a matching of $K_{s, s}$ with $0 \leq|M| \leq s$. By Claim 3, if $G$ has a 3-cycle and $G$ is not $C_{3}$, then $G$ has a 4-cycle, unless $G$ is $G_{1}$. Now we assume that $G \notin\left\{C_{4}, C_{5}, C_{6}, G_{1}, K_{s, s}-M\right\}$, hence that $G$ has a $C_{3}$ and a $C_{4}$. Next we will show that $G$ is pancyclic by proving the following fact.

Fact If $G$ has a $k$-cycle $(k=4,5, \ldots, n-1)$, then $G$ has a $(k+1)$-cycle.

Proof Suppose, by contradiction, that $G$ has a $k$-cycle but no $(k+1)$-cycle for some $k \in\{4,5, \ldots, k-1\}$. Let $C=v_{1} v_{2} \ldots v_{k} v_{1}$ be a $k$-cycle, and let $H$ be a component of $G-V(C)$. Since $G$ is 1-tough, $H$ has at least two neighbors on $C$. We distinguish the cases that $H$ is trivial, i.e., $|V(H)|=1$, and that all components of $G-V(C)$ contain at least one edge.

Case 1. $H$ is trivial.

Suppose that $H=\{w\}$. Denote $N_{C}(w)=\left\{u_{1}, u_{2}, \ldots, u_{s}\right\}$, with $s \geq 2$, the vertices $u_{i}$ chosen in this order according to the orientation of $C$, and taking $u_{s+1}=u_{1}$. Clearly, $u_{i+1} \neq u_{i}^{+}$for any $i \in\{1,2, \ldots, s\}$; otherwise $G$ contains a $(k+1)$-cycle, a contradiction. Now, the $s$ neighbors of $H$ on $C$ divide the cycle $C$ into $s$ segments. Let $S_{i}$ be the segment of $C$ from $u_{i}^{+}$to $u_{i+1}^{-}$, denoted as $S_{i}=x_{i_{1}} x_{i_{2}} \ldots x_{i_{r_{i}}}$. We again prove a number of claims.

Claim 4 For any $i \in\{1,2, \ldots, s\}, r_{i}$ is odd, and $x_{1_{1}} x_{i_{j}} \notin E(G)$ for every odd $j$, and $x_{1_{1}} x_{i_{j}} \in E(G)$ for every even $j$. 
Proof We divide the proof into two cases according to the length of the segment $S_{1}$. Case A. $\left|S_{1}\right|=1$. In this case, $S_{1}=x_{1_{1}}$, and the claim holds for segment $S_{1}$ itself. For any segment $S_{i}(i=2,3, \ldots, s)$, we have that if $x_{1_{1}} x_{i_{j}} \in E(G)$, then $x_{1_{1}} x_{i_{j+1}} \notin E(G)$; otherwise, suppose that $x_{1_{1}} x_{i_{j}} \in E(G)$ and $x_{1_{1}} x_{i_{j+1}} \in E(G)$. Then there is a $(k+1)$-cycle $u_{1} w u_{2} C x_{i_{j}} x_{1_{1}} x_{i_{j+1}} C u_{1}$, a contradiction. If $x_{1_{1}} x_{i_{j}} \notin E(G)$, then $x_{1_{1}} x_{i_{j+1}} \in E(G)$; otherwise, suppose that $x_{1_{1}} x_{i_{j}} \notin E(G)$ and $x_{1_{1}} x_{i_{j+1}} \notin E(G)$. Then $\left\{w, x_{1_{1}}, x_{i_{j}}, x_{i_{j+1}}\right\} \cong 2 K_{1} \cup K_{2}$, a contradiction. For the first vertex $x_{i_{1}}$ and the last vertex $x_{i_{r_{i}}}$ of a segment, we have $x_{1_{1}} x_{i_{1}} \notin E(G)$ and $x_{1_{1}} x_{i_{r_{i}}} \notin E(G)$; otherwise, $u_{1} w u_{i} \bar{C} x_{1_{1}} x_{i_{1}} C u_{1}$ or $u_{2} w u_{i+1} C x_{1_{1}} x_{i_{r_{i}}} \bar{C} u_{2}$ is a $(k+1)$-cycle, a contradiction. Thus, the neighbors of $x_{1_{1}}$ occur alternately along the cycle on every segment $S_{i}$, and the two end vertices of $S_{i}$ are not its neighbor. Hence, $r_{i}$ is odd, and $x_{1_{1}} x_{i_{j}} \notin E(G)$ for every odd $j$, and $x_{1_{1}} x_{i_{j}} \in E(G)$ for every even $j$.

Case B. $\left|S_{1}\right| \geq 2$. First, we deal with the segment $S_{1}$. We have that $x_{1_{1}} x_{2_{1}} \notin E(G)$; otherwise, $u_{1} w u_{2} \bar{C} x_{1_{1}} x_{2_{1}} C u_{1}$ is a $(k+1)$-cycle, a contradiction. We also have that $x_{1_{2}} x_{2_{1}} \in E(G)$; otherwise, $\left\{w, x_{2_{1}}, x_{1_{1}}, x_{1_{2}}\right\} \cong 2 K_{1} \cup K_{2}$, a contradiction. If $x_{1_{1}} x_{1_{j}} \in$ $E(G)$ and $x_{1_{1}} x_{1_{j+1}} \in E(G)$, then we have a $(k+1)$-cycle $u_{1} w u_{2} \bar{C} x_{1_{j+1}} x_{1_{1}} x_{1_{j}}$ $\bar{C} x_{1_{2}} x_{2_{1}} C u_{1}$, a contradiction. If $x_{1_{1}} x_{1_{j}} \notin E(G)$ and $x_{1_{1}} x_{1_{j+1}} \notin E(G)$, then $\left\{w, x_{1_{1}}, x_{1_{j}}, x_{1_{j+1}}\right\} \cong 2 K_{1} \cup K_{2}$, a contradiction. For the last vertex $x_{1_{r_{1}}}$ of $S_{1}$, we will show that it is not a neighbor of $x_{1_{1}}$. Suppose that $\left|S_{2}\right| \geq 2$. We have that $x_{1_{1}} x_{2_{2}} \in E(G)$; otherwise, $\left\{w, x_{1_{1}}, x_{2_{1}}, x_{2_{2}}\right\} \cong 2 K_{1} \cup K_{2}$, a contradiction. If $x_{1_{1}} x_{1_{r_{1}}} \in E(G)$, then $u_{1} w u_{2} x_{2_{1}} x_{1_{2}} C x_{1_{r_{1}}} x_{1_{1}} x_{2_{2}} C u_{1}$ is a $(k+1)$-cycle, a contradiction. Suppose that $\left|S_{2}\right|=1$. If $x_{1_{1}} x_{1_{r_{1}}} \in E(G)$, then $u_{2} w u_{3} C x_{1_{1}} x_{1_{r_{1}}} \bar{C} x_{1_{2}} x_{2_{1}} u_{2}$ is a $(k+1)$ cycle, a contradiction. Hence, $x_{1_{1}} x_{1_{r_{1}}} \notin E(G)$. Then the neighbors of $x_{1_{1}}$ on segment $S_{1}$ occur alternately along the cycle, and the first vertex and the last vertex of $S_{1}$ are not its neighbor. Therefor, $r_{1}$ is odd and the claim holds for segment $S_{1}$.

Next, we consider the other segments $S_{i}(i=2,3, \ldots, s)$. Similar with $x_{2_{1}}$, for $x_{i_{1}}$ we have that $x_{1_{1}} x_{i_{1}} \notin E(G)$. Also, we have that $x_{1_{2}} x_{i_{1}} \in E(G)$; otherwise, $\left\{w, x_{i_{1}}, x_{1_{1}}, x_{1_{2}}\right\} \cong 2 K_{1} \cup K_{2}$, a contradiction. If $x_{1_{1}} x_{i_{j}} \in E(G)$ and $x_{1_{1}} x_{i_{j+1}} \in E(G)$, then we have a $(k+1)$-cycle $u_{1} w u_{i} \bar{C} x_{1_{2}} x_{i_{1}} C x_{i_{j}} x_{1_{1}} x_{i_{j+1}} C u_{1}$, a contradiction. If $x_{1_{1}} x_{i_{j}} \notin E(G)$ and $x_{1_{1}} x_{i_{j+1}} \notin E(G)$, then $\left\{w, x_{1_{1}}, x_{i_{j}}, x_{i_{j+1}}\right\} \cong 2 K_{1} \cup K_{2}$, a contradiction. Thus the neighbors of $x_{1_{1}}$ occur alternately on every segment $S_{i}$ along the cycle. Moreover, $x_{1_{1}} x_{i_{r_{i}}} \notin E(G)$; otherwise, we have a $(k+1)$-cycle $u_{i} w u_{i+1} C x_{1_{1}} x_{i_{r_{i}}} \bar{C} x_{i_{1}} x_{1_{2}} C u_{i}$, a contradiction. Hence, $r_{i}$ is odd, and $x_{1_{1}} x_{i_{j}} \notin E(G)$ for every odd $j$, and $x_{1_{1}} x_{i_{j}} \in E(G)$ for every even $j$.

Denote $W=N_{C}(w)$ and $A=N_{C}\left(x_{1_{1}}\right) \backslash W$. By Claim 4, $|W|+|A|=\frac{|V(C)|}{2}$. If $N\left(x_{1_{1}}\right)=N_{C}\left(x_{1_{1}}\right)$, then $A \cup W$ is a cut set and $G-(A \cup W)$ generates at least three components, including two trivial components with vertex sets $\{w\}$ and $\left\{x_{1_{1}}\right\}$. Since $G$ is $2 K_{1} \cup K_{2}$-free, all the other components are also trivial. Then we get $\frac{|V(C)|}{2}+1$ components by deleting $\frac{|V(C)|}{2}$ vertices, contradicting the fact that $G$ is 1-tough. Hence, we next assume that $x_{1_{1}}$ has a neighbor outside the cycle $C$. Suppose that $y$ is a neighbor of $x_{1_{1}}$ in $G-V(C)$. Then $y \neq w$ and $y w \notin E(G)$. Denote 
$B=V(C) \backslash(W \cup A)$. Before continuing the proof for Case 1, we first prove three more claims.

Claim $5 \quad B \subseteq N(y), A \cap N(y)=\emptyset, W \cap N(y)=\emptyset$ and $A, B$, and $W$ are independent sets.

Proof By the definition of $B$, we have $B \cap N\left(x_{1_{1}}\right)=\emptyset$. If there is a vertex $v_{i} \in B$ such that by $\notin E(G)$, then $\left\{w, v_{i}, x_{1_{1}}, y\right\} \cong 2 K_{1} \cup K_{2}$, a contradiction. Hence, $B \subseteq N(y)$. Suppose that $v_{j} \in A$ and $v_{j} y \in E(G)$. Since $v_{j}^{+} \in B, v_{j}^{+} y \in E(G)$, and there is a $(k+1)$-cycle, a contradiction. For any vertex $v_{i} \in W, v_{i}^{-}, v_{i}^{+} \in B$, hence $v_{i}^{-} y, v_{i}^{+} y \in E(G)$. If $v_{i} y \in E(G)$, then there is a $(k+1)$-cycle, a contradiction. Hence, $A \cap N(y)=\emptyset$ and $W \cap N(y)=\emptyset$.

If there is an edge $v_{i} v_{j}$ in $A$, then $\left\{w, y, v_{i}, v_{j}\right\} \cong 2 K_{1} \cup K_{2}$, a contradiction. If there is an edge $v_{i} v_{j}$ in $B$, then $\left\{w, x_{1_{1}}, v_{i}, v_{j}\right\} \cong 2 K_{1} \cup K_{2}$, a contradiction. Suppose $v_{i}, v_{j} \in W \quad$ and $v_{i} v_{j} \in E(G)$. Since $v_{i}^{-}, v_{j}^{-} \in B, \quad v_{i}^{-} y, v_{j}^{-} y \in E(G)$. Then $v_{i}^{-} y v_{j}^{-} \bar{C} v_{i} v_{j} C v_{i}^{-}$is a $(k+1)$-cycle, a contradiction. Hence, $A, B$, and $W$ are independent sets.

Claim $6 A=\emptyset$.

Proof Suppose, by contradiction, that $A \neq \emptyset$. We claim that every vertex of $A$ is adjacent to every vertex of $W$; otherwise, suppose that $v_{i} \in A, v_{j} \in W$ and $v_{i} v_{j} \notin E(G)$. Then, using Claim 5 we have that $\left\{y, v_{i}, v_{j}, w\right\} \cong 2 K_{1} \cup K_{2}$, a contradiction. Suppose that $v_{i} \in A$ and $v_{j} \in W$, with $v_{i} v_{j} \in E(G)$. Since $v_{i}^{+}, v_{j}^{+} \in B$, by Claim $5, v_{i}^{+} y \in E(G), v_{j}^{+} y \in E(G)$. Then there is a $(k+1)$-cycle $v_{i}^{+} y v_{j}^{+} C v_{i} v_{j} \bar{C} v_{i}^{+}$, a contradiction.

By Claim 6, $S_{i}=x_{i_{1}}$ for every $i \in\{1,2, \ldots, s\}$, and $V(C)=B \cup W,|B|=|W|$. By Claim 5, we have that $B$ and $W$ are independent sets and $B \subseteq N(y), W=N(w), W \cap N(y)=\emptyset$.

Claim $7 N_{G-V(C)}(C)=\{w, y\}$.

Proof Suppose, by contradiction, that $z \in N_{G-V(C)}(C) \backslash\{w, y\}$. If $z v_{i} \in E(G)$ and $v_{i} \in B$, then $y z \notin E(G)$; otherwise, $v_{i} z y v_{i}^{+2} C v_{i}$ is a $(k+1)$-cycle, a contradiction. Then, $\left\{y, z, v_{i}^{+}, w\right\} \cong 2 K_{1} \cup K_{2}$, a contradiction. Now suppose that $z v_{i} \in E(G)$ and $v_{i} \in W$. We have that $y z \in E(G)$; otherwise, $\left\{w, z, y, v_{i}^{+}\right\} \cong 2 K_{1} \cup K_{2}$, a contradiction. If there is another vertex $z^{\prime} \in N_{G-V(C)}(C) \backslash\{w, y, z\}$, using the same arguments, we have that $N_{C}\left(z^{\prime}\right) \subseteq W$ and $z^{\prime} y \in E(G), z^{\prime} w \notin E(G)$. That means, if $N_{G-V(C)}(C) \neq\{w, y\}$, then every vertex $a \in N_{G-V(C)}(C) \backslash\{w, y\}$ has the properties: ay $\in E(G)$, aw $\notin E(G)$ and $N_{C}(a) \subseteq W$. By deleting the vertices of $W \cup\{y\}$ we obtain at least $|B|+2$ components, contradicting the fact that $G$ is 1-tough.

Now, we are ready to complete the proof for Case 1 . By Claim 7, the component that contains $y$ is trivial; otherwise $y$ is a cut vertex. Thus, $G-V(C)$ has precisely two trivial components $\{w\}$ and $\{y\}$. Since $k \geq 4,|B|=|W| \geq 2$. If there are two vertices $v_{i}, v_{j} \in W$ that are not adjacent to a vertex $v_{k} \in B$, then 
$\left\{v_{i}, v_{j}, v_{k}, y\right\} \cong 2 K_{1} \cup K_{2}$, a contradiction. If there are two vertices $v_{i}, v_{j} \in B$ that are not adjacent to a vertex $v_{k} \in W$, then $\left\{v_{i}, v_{j}, v_{k}, w\right\} \cong 2 K_{1} \cup K_{2}$, a contradiction. Thus, each vertex of $W$ has at least $|B|-1$ neighbors in $B$ and each vertex of $B$ has at least $|W|-1$ neighbors in $W$. Hence, $G$ is a balanced bipartite graph $K_{s, s}-M$ with two vertex sets $\{w\} \cup B$ and $\{y\} \cup W$, where $s \geq 3$ and $M$ is a matching of $K_{s, s}$. Since wy $\notin E(G), 1 \leq|M| \leq s$. This contradicts the assumption and completes the proof for Case 1 .

Case 2. All the components of $G-V(C)$ contain at least one edge.

Suppose that $H$ is a component of $G-V(C)$. We distinguish three subcases according to the distribution of $N_{C}(H)$, in particular whether there are nontrivial segments (containing at least one nonneighbor of $H$ between two subsequent neighbors of $H$ on $C$ ) or not. We start with the subcase that there are at least two such segments.

Case 2.1. There are at least two nontrivial segments on $C$.

For a vertex $v_{i} \in V(C)$, if $v_{i} \notin N_{C}(H)$ and $v_{i}^{-} \in N_{C}(H)$, then we say $v_{i}$ is a break vertex. By the assumption, there are at least two break vertices on $C$. We call any two break vertices a break pair. Let $S$ denote the set of all break vertices, and call $S$ the break set (of $C$ ). We use the shorthand $S$ is complete to indicate that $S$ induces a complete graph in $G$. Suppose $a b$ is an edge of $H$. We next prove four claims.

Claim $8 S$ is complete, $V(H)=\{a, b\}$, and $V(C) \backslash S \subseteq N(a) \cup N(b)$, hence $S=V(C) \backslash N_{C}(H)$. Moreover, for any two subsequent break vertices $v_{i}, v_{j} \in S$, $\left|v_{i}^{+} C v_{j}^{-}\right|$is even, and the vertices of $v_{i}^{+} C v_{j}^{-}$are alternately neighbors of $a$ and $b$.

Proof First, we have that $V(C) \backslash N_{C}(H)$ induces a complete graph; otherwise, suppose that $v_{i}, v_{j}$ are two nonadjacent vertices of $V(C) \backslash N_{C}(H)$. Then $\left\{v_{i}, v_{j}, a, b\right\} \cong 2 K_{1} \cup K_{2}, \quad$ a contradiction. Hence, $S$ is complete, since $S \subseteq V(C) \backslash N_{C}(H)$. Also, $H$ is a complete graph; otherwise, suppose that $w_{1}, w_{2}$ are two nonadjacent vertices of $H$, and $v_{i}, v_{j}$ are two vertices of $V(C) \backslash N_{C}(H)$. Then $\left\{w_{1}, w_{2}, v_{i}, v_{j}\right\} \cong 2 K_{1} \cup K_{2}$, a contradiction.

Suppose that $\left\{v_{i}, v_{j}\right\}$ is a break pair and in the segment $v_{i}^{+} C v_{j}^{-}$there is no other break vertex. Since $v_{i}, v_{j} \in S, v_{i} v_{j} \in E(G)$. We have that $N_{H}\left(v_{i}^{-}\right) \cap N_{H}\left(v_{j}^{-}\right)=\emptyset$; otherwise, let $w \in N_{H}\left(v_{i}^{-}\right) \cap N_{H}\left(v_{j}^{-}\right)$. Then $v_{i}^{-} w v_{j}^{-} \bar{C} v_{i} v_{j} C v_{i}^{-}$is a $(k+1)$-cycle, a contradiction. Since $H$ is complete, without loss of generality, we assume that $v_{i}^{-} a \in E(G)$ and $v_{j}^{-} b \in E(G)$. Then $v_{i}^{+} \neq v_{j}^{-}$; otherwise, $v_{i}^{-} a b v_{j}^{-} C v_{i}^{-}$is a $(k+1)$ cycle, a contradiction. If $v_{i}^{+} \notin N_{C}(H)$, then $v_{i}^{+} v_{j} \in E(C)$, and $v_{i}^{-} a b v_{j}^{-} \bar{C} v_{i}^{+} v_{j} C v_{i}^{-}$is a $(k+1)$-cycle, a contradiction. Hence, $v_{i}^{+} \in N_{C}(H)$. We have that $N_{H}\left(v_{i}^{+}\right)=\{a\}$; otherwise, suppose that $v_{i}^{+} c \in E(G)$ and $c \in V(H) \backslash\{a\}$. Then $v_{i}^{-} a c v_{i}^{+} C v_{i}^{-}$is a $(k+1)$-cycle, a contradiction. Since $v_{i}^{+2}$ is not a break vertex and $v_{i}^{-}, v_{i}^{+} \in N(a)$, we have $v_{i}^{+2} \in N(b)$; otherwise, $\left\{v_{i}, v_{i}^{+2}, a, b\right\} \cong 2 K_{1} \cup K_{2}$, a contradiction. If $V(H) \neq\{a, b\}$, suppose $c \in V(H) \backslash\{a, b\}$. Then $v_{i}^{-} a c b v_{i}^{+2} C v_{i}^{-}$is a $(k+1)$-cycle, a contradiction. Hence, $V(H)=\{a, b\}$.

Since $v_{i}^{+} C v_{j}^{-}$contains no other break vertex, $v_{i}^{+} C v_{j}^{-} \subseteq N_{C}(H)=N(a) \cup N(b)$. Since $G$ has no $(k+1)$-cycle, the vertices of $v_{i}^{+} C v_{j}^{-}$are alternately neighbors of $a$ 
and $b$. And since the two end vertices of $v_{i}^{+} C v_{j}^{-}$belong to different neighbor sets of $a$ and $b,\left|v_{i}^{+} C v_{j}^{-}\right|$is even.

By the definition of break vertex, any two break vertices are not consecutive vertices on $C$. Thus the break vertices divide the cycle into segments, and from the above analysis we know that each segment (between two break vertices, and not containing any break vertices) belongs to the union of the neighbor sets of $a$ and $b$. Hence, $V(C) \backslash S \subseteq N(a) \cup N(b)$, and therefore, $S=V(C) \backslash N_{C}(H)$.

Claim $9|S|=2$.

Proof Suppose, by contradiction, that $|S| \geq 3$. Assume that $v_{i}, v_{j}, v_{k} \in S$. By Claim $8, S$ is complete. Thus, $v_{i} v_{j}, v_{i} v_{k}, v_{j} v_{k} \in E(G)$. Since $V(H)=\{a, b\}$, two of the three vertices $v_{i}^{-}, v_{j}^{-}, v_{k}^{-}$have a common neighbor in $\{a, b\}$. Without loss of generality, assume that $v_{i}^{-}, v_{j}^{-} \in N(a)$. Then $v_{i}^{-} a v_{j}^{-} \bar{C} v_{i} v_{j} C v_{i}^{-}$is a $(k+1)$-cycle, a contradiction.

Suppose that $S=\left\{v_{s}, v_{t}\right\}$. By Claim 8, all vertices of $V(C) \backslash\left\{v_{s}, v_{t}\right\}$ are adjacent to $a$ or $b$ alternately, $v_{s}^{-}, v_{s}^{+}$share the same neighbor in $\{a, b\}$, while $v_{s}^{+}, v_{t}^{-}$have different neighbors in $\{a, b\}$. Thus, we have that either $v_{s}^{-}, v_{s}^{+} \in N(a)$ and $v_{t}^{-}, v_{t}^{+} \in N(b)$, or $v_{s}^{-}, v_{s}^{+} \in N(b)$ and $v_{t}^{-}, v_{t}^{+} \in N(a)$. Without loss of generality, we assume that $v_{s}^{-}, v_{s}^{+} \in N(a)$ and $v_{t}^{-}, v_{t}^{+} \in N(b)$. Let $A=N_{C}(a), B=N_{C}(b)$. Clearly, $A \cap B=\emptyset,|A|=|B|$ and $V(C)=A \cup B \cup S$.

Claim $10 A \cup\left\{v_{t}\right\}$ and $B \cup\left\{v_{s}\right\}$ are independent sets.

Proof First, we prove that $A$ and $B$ are independent sets. Suppose that $v_{i}, v_{j} \in A$ and $v_{i} v_{j} \in E(G)$. If $\left\{v_{i}, v_{j}\right\}=\left\{v_{s}^{-}, v_{s}^{+}\right\}$, then $v_{s}^{+} a b v_{s}^{+2} C v_{s}^{-} v_{s}^{+}$is a $(k+1)$-cycle, a contradiction. If $\left\{v_{i}, v_{j}\right\} \neq\left\{v_{s}^{-}, v_{s}^{+}\right\}$, then either $v_{i}^{+}, v_{j}^{+} \in B$ or $v_{i}^{-}, v_{j}^{-} \in B$. Without loss of generality, assume that $v_{i}^{+}, v_{j}^{+} \in B$. Then $v_{i}^{+} b v_{j}^{+} C v_{i} v_{j} \bar{C} v_{i}^{+}$is a $(k+1)$-cycle, a contradiction. Hence, $A$ is independent. Similarly, $B$ is also independent.

Next, we prove that $N\left(v_{t}\right) \cap A=\emptyset$ and $N\left(v_{s}\right) \cap B=\emptyset$. Suppose that $v_{i} \in A$ and $v_{i} v_{t} \in E(G)$. Clearly, either $v_{i}^{-} \in B$ or $v_{i}^{+} \in B$. Without loss of generality, assume $v_{i}^{-} \in B$. Then we have a $(k+1)$-cycle $v_{t}^{-} b v_{i}^{-} \bar{C} v_{t} v_{i} C v_{t}^{-}$, a contradiction. Hence, $N\left(v_{t}\right) \cap A=\emptyset$. Similarly, $N\left(v_{s}\right) \cap B=\emptyset$.

Claim $11 G-V(C)=H$.

Proof Suppose that $w \in V(G) \backslash(V(C) \cup V(H))$. We have that $w v_{s}, w v_{t} \in E(G)$; otherwise, suppose that $w v_{s} \notin E(G)$. Then $\left\{w, v_{s}, a, b\right\} \cong 2 K_{1} \cup K_{2}$, a contradiction. Since $G$ has no $(k+1)$-cycle, $v_{s}^{-}, v_{s}^{+}, v_{t}^{-}, v_{t}^{+} \notin N(w)$. Using Claim 10, $\left\{v_{t}^{-}, v_{t}^{+}, v_{s}, w\right\} \cong 2 K_{1} \cup K_{2}$, a contradiction.

For a vertex $v_{i} \in A$, if $v_{i} v_{s} \notin E(G)$, then $\left\{v_{i}, b, v_{s}, v_{t}\right\} \cong 2 K_{1} \cup K_{2}$, a contradiction. Hence, for each vertex $v_{i} \in A, v_{i} v_{s} \in E(G)$. Similarly, for each vertex $v_{j} \in B$, $v_{j} v_{t} \in E(G)$. If there are two vertices $v_{i}, v_{j} \in B$ that are not adjacent to a vertex $v_{k} \in A$, then $\left\{v_{i}, v_{j}, v_{k}, a\right\} \cong 2 K_{1} \cup K_{2}$, a contradiction. Hence, every vertex of $A$ has at least $|B|-1$ neighbors in $B$. Similarly, every vertex of $B$ has at least $|A|-1$ 
neighbors in $A$. Let $A^{\prime}=A \cup\left\{v_{t}\right\} \cup\{b\}$ and $B^{\prime}=B \cup\left\{v_{s}\right\} \cup\{a\}$. From the above analysis, we conclude that $G=K_{p, p}-M$ for the two independent vertex set $A^{\prime}$ and $B^{\prime}$, and a matching $M$ of $K_{p, p}$. Since $v_{s} b, v_{t} a \notin E(G), 2 \leq|M| \leq p$. This final contradiction to the assumption completes the proof for Case 2.1.

Case 2.2. There is precisely one nontrivial segment on $C$.

In this subcase, there exist two vertices $v_{i}, v_{j}$ on $C$ such that $v_{i} C v_{j} \subseteq N_{C}(H)$ and $v_{j}^{+} C v_{i}^{-} \cap N_{C}(H)=\emptyset$. Let $P=v_{j}^{+} C v_{i}^{-}$, and let $a b$ be an edge of $H$. We again start by proving several claims and considering some subcases.

Claim $12|V(P)| \leq 2$.

Proof Suppose, by contradiction, that $|V(P)| \geq 3$. We have that $\langle V(P)\rangle$ is complete; otherwise, any two nonadjacent vertices of $P$ together with the edge $a b$ will induce a $2 K_{1} \cup K_{2}$. Similarly, $H$ is complete; otherwise, two nonadjacent vertices of $H$ together with an edge of $P$ will induce a $2 K_{1} \cup K_{2}$, a contradiction. Let $v_{k}, v_{k}^{+}$be two consecutive vertices of $N_{C}(H)$, and $w_{1}, w_{2}$ be their neighbors in $H$, respectively. Then $w_{1} w_{2} \in E(G)$ and we get a $(k+1)$-cycle $v_{k} w_{1} w_{2} v_{k}^{+} C v_{j}^{+} v_{j}^{+3} C v_{k}$, a contradiction.

We distinguish the subcases that $|V(P)|=2$ and $|V(P)|=1$.

Case 2.2.1. $|V(P)|=2$, i.e., $P=v_{j}^{+} v_{i}^{-}$.

For convenience, we denote $C=v_{1} v_{2} \ldots v_{k} v_{1}$, and $N_{C}(H)=v_{1} C v_{k-2}$. Then $P=v_{k-1} v_{k}$. In this case, $H$ is complete; otherwise, any two nonadjacent vertices of $H$ together with the edge of $P$ will induce a $2 K_{1} \cup K_{2}$, a contradiction.

Claim $13 V(H)=\{a, b\}$ and $k$ is even.

Proof If $v_{1}$ and $v_{k-2}$ have two different neighbors in $H$, without loss of generality, assume that $v_{1} a, v_{k-2} b \in E(G)$. Then $V(H)=\{a, b\}$; otherwise, suppose $c \in V(H) \backslash\{a, b\}$. Then $v_{1} a c b v_{k-2} \bar{C} v_{1}$ is a $(k+1)$-cycle, a contradiction. Suppose that $N_{H}\left(v_{1}\right)=N_{H}\left(v_{k-2}\right)=\{a\}$. If $v_{1} v_{k-2} \in E(G)$, then $V(H)=\{a, b\}$; otherwise, suppose that $c \in V(H) \backslash\{a, b\}$, and without loss of generality, suppose $v_{2} b \in E(G)$. Then $v_{1} a c b v_{2} C v_{k-2} v_{1}$ is a $(k+1)$-cycle, a contradiction. If $v_{1} v_{k-2} \notin E(G)$, then $V(H)=\{a, b\} ; \quad$ otherwise, for any vertex $c \in V(H) \backslash\{a, b\}$ we have $\left\{v_{1}, v_{k-2}, b, c\right\} \cong 2 K_{1} \cup K_{2}, \quad$ a contradiction. Hence, $\quad V(H)=\{a, b\} \quad$ and $V(C) \backslash V(P) \subseteq N(a) \cup N(b)$. Since $G$ has no $(k+1)$-cycle, each vertex of $V(C) \backslash V(P)$ is adjacent to one and only one of $\{a, b\}$, alternately. If we can prove that $v_{1}, v_{k-2}$ are adjacent to different vertices of $\{a, b\}$, then we get that $k$ is even. First, we have that $v_{k} v_{2} \notin E(G)$ and $v_{k-3} v_{k-1} \notin E(G)$; otherwise, suppose $v_{k} v_{2} \in E(G)$. Then $v_{2} b a v_{3} C v_{k} v_{2}$ (if $k \geq 5$ ) or $v_{k} v_{2} b a v_{1} v_{k}$ (if $k=4$ ) is a $(k+1)$ cycle, a contradiction. Suppose that $v_{1}, v_{k-2}$ are adjacent to the same vertex of $\{a, b\}$, say $a$ without loss of generality. Clearly, $v_{2} v_{k-1} \notin E(G)$. Then $\left\{a, v_{2}, v_{k-1}, v_{k}\right\} \cong 2 K_{1} \cup K_{2}$, a contradiction. Hence, $v_{1}, v_{k-2}$ are adjacent to different vertices of $\{a, b\}$, and $|V(C) \backslash V(P)|$ is even, so $k$ is even.

Let $A=N_{C}(a)=\left\{v_{1}, v_{3}, \ldots, v_{k-3}\right\}, \quad B=N_{C}(b)=\left\{v_{2}, v_{4}, \ldots, v_{k-2}\right\} . \quad$ Then $V(C)=A \cup B \cup V(P), A \cap B=\emptyset$ and $|A|=|B|$. 
Claim $14 G-V(C)=H$.

Proof Suppose, by contradiction, that $w \in V(G) \backslash(V(C) \cup V(H))$. To avoid $\left\{w, v_{k-1}, a, b\right\} \quad$ or $\quad\left\{w, v_{k}, a, b\right\} \quad$ inducing $2 K_{1} \cup K_{2}$, we have that $w v_{k-1}, w v_{k} \in E(G)$. Then there clearly is a $(k+1)$-cycle, a contradiction.

Claim $15 A \cup\left\{v_{k-1}\right\}$ and $B \cup\left\{v_{k}\right\}$ are independent sets.

Proof First, we prove that $A$ and $B$ are independent sets. If $|A|=|B|=1$, then the claim holds. Now we suppose that $|A|=|B| \geq 2$. If $v_{i}, v_{j} \in A$ and $v_{i} v_{j} \in E(G)$, then either $v_{i}^{-}, v_{j}^{-} \in B$ or $v_{i}^{+}, v_{j}^{+} \in B$. Without loss of generality, assume $v_{i}^{-}, v_{j}^{-} \in B$. Then there is a $(k+1)$-cycle $v_{i}^{-} b v_{j}^{-} \bar{C} v_{i} v_{j} C v_{i}^{-}$, a contradiction. Hence, $A$ is independent set. Similarly, $B$ is also independent set.

Next, we prove that $N\left(v_{k-1}\right) \cap A=\emptyset$ and $N\left(v_{k}\right) \cap B=\emptyset$. Suppose that $v_{i} \in A$ and $v_{i} v_{k-1} \in E(G)$. If $v_{i}=v_{1}$, then $v_{k-1} v_{1} a b v_{2} C v_{k-1}$ is a $(k+1)$-cycle. If $v_{i} \neq v_{1}$, then $v_{i}^{-} \in B$ and $v_{i}^{-} b v_{k-2} \bar{C} v_{i} v_{k-1} C v_{i}^{-}$is a $(k+1)$-cycle, a contradiction. Hence, $N\left(v_{k-1}\right) \cap A=\emptyset$. Similarly, $N\left(v_{k}\right) \cap B=\emptyset$.

If there is a vertex $v_{i} \in A$ such that $v_{i} v_{k} \notin E(G)$, then $v_{i} \neq v_{1}$. Since $v_{1} \in A$, $v_{i} v_{1} \notin E(G)$. Then $\left\{b, v_{i}, v_{k}, v_{1}\right\} \cong 2 K_{1} \cup K_{2}$, a contradiction. Hence, $A \subseteq N\left(v_{k}\right)$. Similarly, $B \subseteq N\left(v_{k-1}\right)$. If there are two vertices $v_{i}, v_{j} \in B$ that are not adjacent to a vertex $v_{s} \in A$, then $\left\{v_{i}, v_{j}, v_{s}, a\right\} \cong 2 K_{1} \cup K_{2}$, a contradiction. Hence, each vertex of $A$ has at least $|B|-1$ neighbors in $B$. Similarly, each vertex of $A$ has at least $|B|-1$ neighbors in $B$. Thus, $G=K_{s, s}-M$ for the two independent vertex sets $A \cup\{b\} \cup$ $\left\{v_{k-1}\right\}$ and $B \cup\{a\} \cup\left\{v_{k}\right\}$, and a matching $M$ of $K_{s, s}$. Since $a v_{k-1} \notin E(G)$ and $b v_{k} \notin E(G), 2 \leq|M| \leq s$. This contradiction to the assumption completes the proof for Case 2.2.1.

Case 2.2.2. $|V(P)|=1$.

Without loss of generality, we assume that $v_{k}$ is the only vertex that has no neighbor in $H$.

Claim $16|V(H)| \geq 3$.

Proof Suppose, by contradiction, that $V(H)=\{a, b\}$. All vertices except $v_{k}$ of $C$ are adjacent to $a$ or $b$. Without loss of generality, assume $v_{1} \in N(a)$. Since $G$ has no $(k+1)$-cycle, $k$ is even, and $N_{C}(a)=\left\{v_{1}, v_{3}, v_{5}, \ldots, k-1\right\}, N_{C}(b)=\left\{v_{2}, v_{4}, v_{6}, \ldots\right.$ $, k-2\}$. Denote $A=N_{C}(a)$ and $B=N_{C}(b)$. Then $V(C)=A \cup B \cup\left\{v_{k}\right\}$, and $A \cap B=\emptyset$.

Claim 16.1 $G-V(C)=H$.

Proof Suppose that $H^{\prime}$ is another component of $G-V(C)$. We have that $V\left(H^{\prime}\right) \subseteq N\left(v_{k}\right)$; otherwise, suppose that $w \in V\left(H^{\prime}\right)$ and $w v_{k} \notin E(G)$. Then $\left\{w, v_{k}, a, b\right\} \cong 2 K_{1} \cup K_{2}$, a contradiction. Now $v_{1}, v_{k-1} \notin N_{C}\left(H^{\prime}\right)$; otherwise there is a $(k+1)$-cycle. Since $k \geq 4$ and $\left|N_{C}\left(H^{\prime}\right)\right| \geq 2$, the neighbors of $H^{\prime}$ on $C$ are not consecutive, and we are in Case 2.1, a contradiction.

Claim 16.2 $A$ and $B$ are independent sets. 
Proof Suppose that $v_{i}, v_{j} \in A$ and $v_{i} v_{j} \in E(G)$. If $\left\{v_{i}, v_{j}\right\}=\left\{v_{1}, v_{k-1}\right\}$, then $v_{1} a b v_{2} C v_{k-1} v_{1}$ is a $(k+1)$-cycle, a contradiction. If $\left\{v_{i}, v_{j}\right\} \neq\left\{v_{1}, v_{k-1}\right\}$, then either $v_{i}^{+}, v_{j}^{+} \in B$ or $v_{i}^{-}, v_{j}^{-} \in B$, say $v_{i}^{+}, v_{j}^{+} \in B$ without loss of generality. Then we have a $(k+1)$-cycle $v_{i}^{+} b v_{j}^{+} C v_{i} v_{j} \bar{C} v_{i}^{+}$, a contradiction. Hence, $A$ is an independent set. If $v_{i}, v_{j} \in B$ and $v_{i} v_{j} \in E(G)$, then $v_{i}^{+}, v_{j}^{+} \in A$ and we get a $(k+1)$-cycle $v_{i}^{+} a v_{j}^{+} C v_{i} v_{j} \bar{C} v_{i}^{+}$, a contradiction. Hence, $B$ is an independent set.

Claim 16.3 $A \subseteq N\left(v_{k}\right)$ and $N\left(v_{k}\right) \cap B=\emptyset$.

Proof Suppose there is a vertex $v_{i} \in A$ such that $v_{i} v_{k} \notin E(G)$. Clearly, $v_{i} \neq v_{1}$, and $v_{i} v_{1} \notin E(G)$ by Claim 16.2. Then $\left\{b, v_{i}, v_{k}, v_{1}\right\} \cong 2 K_{1} \cup K_{2}$, a contradiction. Hence, $A \subseteq N\left(v_{k}\right)$. If $v_{j} \in B$ such that $v_{j} v_{k} \in E(G)$, then $v_{j}^{-} \in A$ and there is a $(k+1)$-cycle $v_{j}^{-} a v_{k-1} \bar{C} v_{j} v_{k} C v_{j}^{-}$, a contradiction. Hence, $N\left(v_{k}\right) \cap B=\emptyset$.

If there are two vertices $v_{i}, v_{j} \in B$ that are not adjacent to a vertex $v_{k} \in A$, then $\left\{v_{i}, v_{j}, v_{k}, a\right\} \cong 2 K_{1} \cup K_{2}$, a contradiction. Similarly, if there are two vertices $v_{i}, v_{j} \in$ $A$ that are not adjacent to a vertex $v_{k} \in B$, then $\left\{v_{i}, v_{j}, v_{k}, b\right\} \cong 2 K_{1} \cup K_{2}$, a contradiction. Hence, every vertex of $A$ has at least $|B|-1$ neighbors in $B$, and every vertex of $B$ has at least $|A|-1$ neighbors in $A$. Thus, $G=K_{s, s}-M$ for the two independent sets $A \cup\{b\}$ and $B \cup\{a\} \cup\left\{v_{k}\right\}$, and a matching $M$ of $K_{s, s}$. Since $b v_{k} \notin E(G)$ and $A \cup\{b\} \subseteq N(a), 1 \leq|M| \leq s-1$. This contradicts the assumption.

\section{Claim $17 \quad H$ is not complete.}

Proof Suppose, by contradiction, that $H$ is complete. Using Claim 16, if $v_{1} w_{1} \in$ $E(G), v_{k-1} w_{2} \in E(G)$ for two distinct vertices $w_{1}, w_{2} \in V(H)$, then $v_{1} w_{1} w_{2} v_{k-1} \bar{C} v_{1}$ is a $(k+1)$-cycle, a contradiction. Hence $v_{1}$ and $v_{k-1}$ have only one common neighbor, say $w_{1}$. We have that $v_{k} v_{k-2} \notin E(G)$; otherwise, $v_{1} C v_{k-2} v_{k} v_{k-1} w_{1} v_{1}$ is a $(k+1)$-cycle, a contradiction. Suppose that $w_{1} w_{2} \in E(H)$. To avoid $\left\{v_{k}, v_{k-2}, w_{1}, w_{2}\right\}$ inducing $2 K_{1} \cup K_{2}$, we have $w_{2} v_{k-2} \in E(G)$. Since $|V(H)| \geq 3$ and $H$ is complete, there is another vertex $w_{3} \in V(H) \backslash\left\{w_{1}, w_{2}\right\}$ and $w_{1} w_{3}, w_{2} w_{3} \in E(G)$. Then $v_{1} C v_{k-2} w_{2} w_{3} w_{1} v_{1}$ is a $(k+1)$-cycle, a contradiction. Hence, $H$ is not complete.

Denote $A=\left\{v_{i} \in V(C) \mid 1 \leq i<k\right.$ and $i$ is odd $\}, B=\left\{v_{i} \in V(C) \mid 2 \leq i<k\right.$ and $i$ is even $\}$, and denote $A_{H}=N_{H}(A)$ and $B_{H}=N_{H}(B)$.

Claim $18 A_{H}$ and $B_{H}$ are independent sets.

Proof It is sufficient if we can prove that for any two vertices $v_{i}, v_{j}(1 \leq i<j<k)$ such that $\left|v_{i} C v_{j}\right|$ is odd, either they share a common neighbor in $H$ or their neighbors in $H$ are nonadjacent. We use induction to prove that fact. First, suppose that $\left|v_{i} C v_{j}\right|=3$. If $v_{i}, v_{j}$ have different neighbors in $H$, say $w_{1}, w_{2}$, respectively, then using Claim 17, $w_{1} w_{2} \in E(H)$. But then $v_{i} w_{1} w_{2} v_{j} C v_{i}$ is a $(k+1)$-cycle, a contradiction. Hence the claim holds for the case $\left|v_{i} C v_{j}\right|=3$. Now suppose that the claim holds for $\left|v_{i} C v_{j}\right| \leq 2 m-1(m \geq 2)$. Then it is sufficient to deal with the 
case $\left|v_{i} C v_{j}\right|=2 m+1$. Suppose that $v_{i} w_{1} \in E(G), v_{j} w_{2} \in E(G)$ and $w_{1} w_{2} \in E(H)$. If $v_{i}^{+2} w_{1} \in E(G)$, then $\left|v_{i}^{+2} C v_{j}\right|=2 m-1$ and $w_{1} w_{2} \in E(H)$, contradicting the assumption. If $v_{i}^{+2} w_{2} \in E(G)$, then $\left|v_{i} C v_{i}^{+2}\right|=3$ and $w_{1} w_{2} \in E(H)$, contradicting the assumption. Hence, $v_{i}^{+2}$ has a neighbor different from $w_{1}, w_{2}$ in $H$, say $w_{3}$, and $w_{1} w_{3}, w_{2} w_{3} \notin E(G)$. To avoid $\left\{w_{3}, v_{i}^{+}, w_{1}, w_{2}\right\}$ inducing $2 K_{1} \cup K_{2}$, we have $v_{i}^{+} w_{2} \in E(G)$. For the vertex $v_{j}^{-}$, by the same arguments we get that $v_{j}^{-} w_{1} \in E(G)$. Then $\left|v_{i}^{+} C v_{j}^{-}\right|=2 m-1$ and $w_{2}, w_{1}$ are their neighbors, respectively. Since $w_{1} w_{2} \in E(H)$, that contradicts the induction hypothesis. Hence, the claim holds for the case $\left|v_{i} C v_{j}\right|=2 m+1$ and the proof is complete.

Claim $19 A_{H} \cap B_{H}=\emptyset$ and $V(H)=A_{H} \cup B_{H}$.

Proof Suppose that there is a vertex $w \in A_{H} \cap B_{H}$. Then $w$ has two neighbors $v_{i}, v_{j}$ on $C$ such that $i$ is odd and $j$ is even. Clearly, $j \neq i+1, j \neq i-1$ and $v_{i}^{+} v_{j}^{+} \notin E(G)$; otherwise there is a $(k+1)$-cycle. By Claim 18, $w$ has no neighbor in $A_{H} \cup B_{H}$. Since $H$ is connected and nontrivial, there is a vertex $w^{\prime} \in V(H) \backslash\left(A_{H} \cup B_{H}\right)$ such that $w w^{\prime} \in E(G)$. Since $N_{H}(C)=A_{H} \cup B_{H}, w^{\prime} v_{i}^{+} \notin E(G)$ and $w^{\prime} v_{j}^{+} \notin E(G)$. Then $\left\{v_{i}^{+}, v_{j}^{+}, w, w^{\prime}\right\} \cong 2 K_{1} \cup K_{2}$, a contradiction. Hence, $A_{H} \cap B_{H}=\emptyset$.

Suppose that $w \in V(H) \backslash\left(A_{H} \cup B_{H}\right)$. Since $\quad N_{H}(C)=A_{H} \cup B_{H}$, $N(w) \cap V(C)=\emptyset$. Assume that $w_{1} \in B_{H}$ and $w_{1} v_{2} \in E(G)$. We have that $w w_{1} \in E(G)$; otherwise, $\left\{w, w_{1}, v_{1}, v_{k}\right\} \cong 2 K_{1} \cup K_{2}$, a contradiction. Assume that $w_{2} v_{k-1} \in E(G)$. We have that $w_{2} \neq w_{1}$; otherwise, $v_{k} v_{3} \notin E(G)$ and $\left\{v_{k}, v_{3}, w_{1}, w\right\} \cong 2 K_{1} \cup K_{2}$, a contradiction. If $w w_{2} \in E(G)$, then we get a $(k+1)$-cycle $v_{2} C v_{k-1} w_{2} w w_{1} v_{2}$, a contradiction. Thus, $w w_{2} \notin E(G)$. To avoid $\left\{w, w_{2}, v_{1}, v_{k}\right\}$ or $\left\{w_{2}, v_{k}, w, w_{1}\right\}$ inducing $2 K_{1} \cup K_{2}$, we have that $w_{2} v_{1} \in E(G)$ and $w_{2} w_{1} \in E(G)$. Thus, $w_{2} \in A_{H}$ and $v_{k-1} \in A$. We have $v_{1} v_{k-1} \notin E(G)$; otherwise, $v_{1} w_{2} w_{1} v_{2} C v_{k-1} v_{1}$ is a $(k+1)$-cycle, a contradiction. Since $A_{H} \cap B_{H}=\emptyset$, $w_{1} v_{k-1} \notin E(G)$. Then $\left\{v_{1}, v_{k-1}, w, w_{1}\right\} \cong 2 K_{1} \cup K_{2}$, a contradiction. Hence, $V(H)=A_{H} \cup B_{H}$.

Claim $20\left|B_{H}\right| \geq 2$.

Proof Suppose, by contradiction, that $B_{H}=\{w\}$. Since $V(H)=A_{H} \cup B_{H}$ and $|V(H)| \geq 3,\left|A_{H}\right| \geq 2$. Suppose first that $k$ is odd. Then $v_{k-1} w \in E(G)$. Suppose that $v_{1} w_{1} \in E(G)$ and $w_{1} \in A_{H}$. We have that $w w_{1} \notin E(G)$; otherwise, $v_{1} C v_{k-1} w w_{1} v_{1}$ is a $(k+1)$-cycle, a contradiction. By Claims 18 and $19, w_{1}$ has no neighbor in $H$, contradicting the fact that $H$ is connected. Hence, $k$ is even. Moreover, we have that $B \cup\left\{v_{k}\right\}$ is an independent set; otherwise, suppose that $v_{i}, v_{j} \in B \cup\left\{v_{k}\right\}$ and $v_{i} v_{j} \in E(G)$. Then $\left\{w_{1}, w_{2}, v_{i}, v_{j}\right\} \cong 2 K_{1} \cup K_{2}$, where $w_{1}, w_{2} \in A_{H}$, a contradiction. Now, if we delete all the vertices of $A \cup\{w\}$, then we will get $\left|B \cup\left\{v_{k}\right\}\right|+\left|A_{H}\right|$ trivial components. Since $\quad\left|B \cup\left\{v_{k}\right\}\right|=|A|$ and $\left|A_{H}\right| \geq 2$, $\left|B \cup\left\{v_{k}\right\}\right|+\left|A_{H}\right|>|A \cup\{w\}|$. This contradicts the fact that $G$ is 1-tough.

By Claims 18-20, there are two vertices $w_{1}, w_{2} \in B_{H}$ such that $w_{1} w_{2} \notin E(G)$ and $w_{1}, w_{2} \notin N\left(v_{1}\right)$. Then $\left\{w_{1}, w_{2}, v_{1}, v_{k}\right\} \cong 2 K_{1} \cup K_{2}$, our final contradiction that completes the proof for Case 2.2. 
Case 2.3. There is no nontrivial segment on $C$.

In this case, all the vertices of $C$ are neighbors of a component $H$ of $G-V(C)$. We first claim that $H$ has at least three vertices; otherwise $G$ belongs to the class of graphs that we have excluded.

Claim $21|V(H)| \geq 3$.

Proof Suppose, by contradiction, that $V(H)=\{a, b\}$. Then all the vertices of $C$ are adjacent to $a$ or $b$. Without loss of generality, assume that $v_{1} a \in E(G)$. Then we have that $k$ is even and $N_{C}(a)=\left\{v_{i} \mid 1 \leq i \leq k\right.$ and $i$ is odd $\}, N_{C}(b)=\left\{v_{j} \mid 1 \leq j \leq k\right.$ and $i$ is even $\}$. Denote $A=N_{C}(a), B=N_{C}(b)$. Then $V(C)=A \cup B$.

Claim 21.1 A and B are independent sets.

Proof Suppose that $v_{i}, v_{j} \in A$. Clearly, $v_{i}^{+}, v_{j}^{+} \in B$. If $v_{i} v_{j} \in E(G)$, then we get a $(k+1)$-cycle $v_{i}^{+} b v_{j}^{+} C v_{i} v_{j} \bar{C} v_{i}^{+}$, a contradiction. Hence, $A$ is independent. Similarly, $B$ is also independent.

Claim 21.2 $G-V(C)=H$ or $V(G) \backslash(V(C) \cup V(H))=\left\{a^{\prime}, b^{\prime}\right\}$. Moreover, in the latter case $A \subseteq N\left(a^{\prime}\right)$ and $B \subseteq N\left(b^{\prime}\right)$.

Proof Since $G$ is $2 K_{1} \cup K_{2}$-free and all components of $G-V(C)$ are nontrivial, $G-V(C)$ has either one component or two complete components. If $G-V(C)$ has one component, then the claim holds. Suppose that there is another component $H^{\prime}=G-(V(C) \cup V(H))$. Then there is a vertex $a^{\prime} \in H^{\prime}$ such that $a^{\prime}$ has a neighbor on $C$. Without loss of generality, assume $a^{\prime} v_{1} \in E(G)$. Then $A \subseteq N\left(a^{\prime}\right)$; otherwise, suppose $v_{i} a^{\prime} \notin E(G)$ and $v_{i} \in A$. Then $\left\{b, v_{i}, a^{\prime}, v_{1}\right\} \cong 2 K_{1} \cup K_{2}$, a contradiction. Hence, $A \subseteq N\left(a^{\prime}\right)$ and $B \cap N\left(a^{\prime}\right)=\emptyset$. Suppose that $a^{\prime} b^{\prime} \in E\left(H^{\prime}\right)$. Then $B \subseteq N\left(b^{\prime}\right)$; otherwise, suppose $v_{i} b^{\prime} \notin E(G)$ and $v_{i} \in B$. Then $\left\{a, v_{i}, a^{\prime}, b^{\prime}\right\} \cong 2 K_{1} \cup K_{2}$, a contradiction. If $V\left(H^{\prime}\right) \neq\left\{a^{\prime}, b^{\prime}\right\}$, assume $c^{\prime} \in V\left(H^{\prime}\right) \backslash\left\{a^{\prime}, b^{\prime}\right\}$. Then there is a $(k+1)$-cycle $v_{1} a^{\prime} c^{\prime} b^{\prime} v_{4} C v_{1}$, a contradiction. Hence, the claim holds.

If there are two vertices $v_{i}, v_{j} \in B$ that are not adjacent to a vertex $v_{k} \in A$, then $\left\{v_{i}, v_{j}, v_{k}, a\right\} \cong 2 K_{1} \cup K_{2}$, a contradiction. Similarly, if there are two vertices $v_{i}, v_{j} \in$ $A$ that are not adjacent to a vertex $v_{k} \in B$, then $\left\{v_{i}, v_{j}, v_{k}, b\right\} \cong 2 K_{1} \cup K_{2}$, a contradiction. Hence, every vertex of $A$ has at least $|B|-1$ neighbors in $B$, and every vertex of $B$ has at least $|A|-1$ neighbors in $A$. Thus, if $G-V(C)=H$, then $G=K_{s, s}-M$ for the two independent sets $A \cup\{b\}$ and $B \cup\{a\}$, and a matching $M$ of $K_{s, s}$. Since $N(a)=A \cup\{b\} \quad$ and $\quad N(b)=B \cup\{a\}, \quad 0 \leq|M| \leq s-2$. If $V(G) \backslash(V(C) \cup V(H))=\left\{a^{\prime}, b^{\prime}\right\}$, then $G=K_{s, s}-M$ for the two independent sets $A \cup\left\{b, b^{\prime}\right\}$ and $B \cup\left\{a, a^{\prime}\right\}$, and a matching $M$ of $K_{s, s}$. Since $a b^{\prime} \notin E(G)$ and $b a^{\prime} \notin E(G), 2 \leq|M| \leq s$. This contradicts the assumption.

Claim $22 H$ is not complete.

Proof Suppose, by contradiction, that $H$ is complete. If $\left|N_{H}(C)\right|=2$ and $N_{H}(C)=\{a, b\}$, then all the vertices of $C$ are neighbors of $a$ or $b$ alternately. Denote $A=N_{C}(a)$ and $B=N_{C}(b)$. Then $|A|=|B| \geq 2$ and $A, B$ are independent sets. Taking two vertices $v_{i}, v_{j}$ from $A$ and a vertex $c$ from $V(H) \backslash\{a, b\}$, we get that 
$\left\{v_{i}, v_{j}, b, c\right\} \cong 2 K_{1} \cup K_{2}$, a contradiction. Hence, $\left|N_{H}(C)\right| \geq 3$. Then there are three vertices $a, b, c \in V(H)$ and a vertex $v_{i} \in V(C)$ such that $a v_{i}, b v_{i}^{+}, c v_{i}^{+2} \in E(G)$. Then we get a $(k+1)$-cycle $v_{i} a c v_{i}^{+2} C v_{i}$, a contradiction. Hence, $H$ is not complete.

Using Claim 22, we get that $G-V(C)=H$; otherwise, suppose $H^{\prime}$ is another component of $G-V(C)$. Then two nonadjacent vertices of $H$ with an edge of $H^{\prime}$ will induce a $2 K_{1} \cup K_{2}$, a contradiction. Denote $A=\left\{v_{i} \in V(C) \mid 1 \leq i \leq k\right.$ and $i$ is odd $\}, B=\left\{v_{i} \in V(C) \mid 2 \leq i \leq k\right.$ and $i$ is even $\}$, and denote $A_{H}=N_{H}(A)$ and $B_{H}=N_{H}(B)$.

Claim $23 A_{H}$ and $B_{H}$ are independent sets.

Proof The only difference between the conditions of Claims 18 and 23 is that $v_{k}$ is a neighbor of $H$ in the latter one but not in the former one. In the induction proof of Claim 18, the absence of $v_{k}$ does not affect the result. Hence, the proof of Claim 18 is also valid here.

Claim $24 \quad V(H)=A_{H} \cup B_{H}$ and $A_{H} \cap B_{H}=\emptyset$.

Proof Suppose, by contradiction, that $V(H) \neq A_{H} \cup B_{H}$. There is a vertex $w \in$ $V(G) \backslash\left(A_{H} \cup B_{H}\right) \quad$ such that $\quad w w_{1} \in E(H)$, where $w_{1} \in A_{H} \cup B_{H}$. Since $N_{H}(C)=A_{H} \cup B_{H}, w$ has no neighbor on $C$. Without loss of generality, assume that $w_{1} \in A_{H}$ and $w_{1} v_{1} \in E(G)$. If $v_{k} v_{2} \notin E(G)$, then $\left\{v_{k}, v_{2}, w_{1}, w\right\} \cong 2 K_{1} \cup K_{2}$, a contradiction. Hence, $v_{k} v_{2} \in E(G)$. Suppose that $v_{2} w_{2}, v_{3} w_{3} \in E(G)$ with $w_{2}, w_{3} \in V(H)$. Obviously, $w_{2} \neq w_{1}, w_{2} \neq w_{3}$, and since $v_{k} v_{2} \in E(G)$ we have $w_{1} \neq w_{3}$ and $w_{1} v_{3}, w_{3} v_{1} \notin E(G)$. By Claim 23, $w_{1} w_{3} \notin E(G)$. Moreover, we have $w_{2} w_{3} \notin E(G)$; otherwise, $v_{2} w_{2} w_{3} v_{3} C v_{k} v_{2}$ is a $(k+1)$-cycle, a contradiction. If $w_{1} w_{2} \notin E(G)$, then $\left\{w_{2}, w_{3}, w_{1}, v_{1}\right\} \cong 2 K_{1} \cup K_{2}$, a contradiction. Hence, $w_{1} w_{2} \in E(G)$. If $k=4$, then we get a $(k+1)$-cycle $v_{1} w_{1} w_{2} v_{2} v_{k} v_{1}$, a contradiction. Thus, $k \geq 5$ and $v_{4} \neq v_{k}$. We have $v_{1} v_{4} \notin E(G)$ and $w_{1} v_{4} \notin E(G)$; otherwise, $v_{k} v_{2} w_{2} w_{1} v_{1} v_{4} C v_{k}$ or $v_{2} w_{2} w_{1} v_{4} C v_{2}$ is a $(k+1)$-cycle, respectively, a contradiction. Then $\left\{w_{3}, v_{4}, w_{1}, v_{1}\right\} \cong 2 K_{1} \cup K_{2}$, a contradiction. Hence, $V(H)=A_{H} \cup B_{H}$.

Suppose that $w \in A_{H} \cap B_{H}$. By Claim 23, $w$ has no neighbor in $A_{H} \cup B_{H}$. Since $V(H)=A_{H} \cup B_{H}, w$ is a independent vertex of $H$, contradicting the fact that $H$ is connected. Hence, $A_{H} \cap B_{H}=\emptyset$.

Claim $25 k$ is even.

Proof For two vertices $v_{i}, v_{j} \in V(C)$, if $i$ and $j$ are both odd or both even, then we say that $v_{i}, v_{j}$ are in the same group, and by Claim 23 we know that their neighbors on $H$ are independent. Suppose that $k$ is odd, and $v_{k} w \in E(G)$ with $w \in V(H)$. Clearly, $w \in A_{H}$. Since $v_{k}^{+2}=v_{2}, v_{k}^{+4}=v_{4}, \ldots$, if we relabel the vertices on $C$ by increasing the subscript of every vertex by one, then $v_{k}$ becomes $v_{1}, v_{2}$ becomes $v_{3}$, $\ldots$. Then the original $v_{k}$ and the original $v_{2}, v_{4}, \ldots$ are in the same group. Then $w$ is independent with every vertex of $B_{H}$. By Claims 23 and 24, $w$ has no neighbor in $H$, contradicting that $H$ is connected. Hence, $k$ is even. 
Claim $26 A$ and $B$ are independent sets.

Proof Suppose that $v_{i}, v_{j} \in A$ and $v_{i} v_{j} \in E(G)$. If $\left|B_{H}\right|=1$, assume $B_{H}=\{w\}$. Then $v_{i}^{+} w, v_{j}^{+} w \in E(G)$ and we get a $(k+1)$-cycle $v_{i}^{+} w v_{j}^{+} C v_{i} v_{j} \bar{C} v_{i}^{+}$, a contradiction. If $\left|B_{H}\right| \geq 2$, assume $w_{1}, w_{2} \in B_{H}$. Then $\left\{w_{1}, w_{2}, v_{i}, v_{j}\right\} \cong 2 K_{1} \cup K_{2}$, a contradiction. Hence, $A$ is an independent set. Similarly, $B$ is also and independent set.

By Claims 23-26, $A \cup B_{H}$ and $B \cup A_{H}$ are independent. Since $G$ is 1-tough and $V(G)=A \cup B \cup A_{H} \cup B_{H}$, we have $\left|A \cup B_{H}\right|=\left|B \cup A_{H}\right|$.

Claim 27 For each vertex $x \in A \cup B_{H}$, $x$ has at least $\left|B \cup A_{H}\right|-1$ neighbors in $B \cup A_{H}$, and for each vertex $y \in B \cup A_{H}$, y has at least $\left|A \cup B_{H}\right|-1$ neighbors in $A \cup B_{H}$.

Proof Suppose that $y_{1}, y_{2}$ are two vertices of $B \cup A_{H}$ such that they are not adjacent to a vertex $x \in A \cup B_{H}$. If $x \in A$ and $y_{1}, y_{2} \in B$, then $\left\{y_{1}, y_{2}, x, w\right\} \cong 2 K_{1} \cup K_{2}$, where $w$ is a neighbor of $x$ in $A_{H}$, a contradiction. If $x \in A$ and $y_{1}, y_{2} \in A_{H}$, then $\left\{y_{1}, y_{2}, x, x^{+}\right\} \cong 2 K_{1} \cup K_{2}$, a contradiction. If $x \in A$ and $y_{1} \in B, y_{2} \in A_{H}$, then there is another vertex $y_{3} \in A_{H} \backslash\left\{y_{2}\right\} \quad$ such that $x y_{3} \in E(G)$, and $\left\{y_{1}, y_{2}, x, y_{3}\right\} \cong 2 K_{1} \cup K_{2}$, a contradiction.

If $x \in B_{H}$ and $y_{1}, y_{2} \in B$, then $\left\{y_{1}, y_{2}, x, z\right\} \cong 2 K_{1} \cup K_{2}$, where $z$ is a neighbor of $x$ in $A_{H}$, a contradiction. If $x \in B_{H}$ and $y_{1}, y_{2} \in A_{H}$, then $\left\{y_{1}, y_{2}, x, z^{\prime}\right\} \cong 2 K_{1} \cup K_{2}$, where $z^{\prime}$ is a neighbor of $x$ in $B$, a contradiction. If $x \in B_{H}$ and $y_{1} \in B, y_{2} \in A_{H}$, then there is another vertex $y_{3}^{\prime} \in B \backslash\left\{y_{1}\right\}$ such that $x y_{3}^{\prime} \in E(G)$, and $\left\{y_{1}, y_{2}, x, y_{3}^{\prime}\right\} \cong 2 K_{1} \cup K_{2}$, a contradiction. Hence, for each vertex $x \in A \cup B_{H}, x$ has at least $\left|B \cup A_{H}\right|-1$ neighbors in $B \cup A_{H}$. By symmetry, for each vertex $y \in B \cup A_{H}, y$ has at least $\left|A \cup B_{H}\right|-1$ neighbors in $A \cup B_{H}$.

By Claim 27, we have that $G=K_{s, s}-M$ for the two independent sets $A \cup B_{H}$ and $B \cup A_{H}$, and a matching $M$ of $K_{s, s}$ with $0 \leq|M| \leq s$. This final contradiction to the assumption completes the proof for Case 2.3, and also completes the proof of Theorem 10.

\section{Proof of Theorem 11}

Before we present our proof of Theorem 11, we state the following lemma to narrow down the category of graphs that we need to consider.

Lemma 1 (Hendry [13]) If $G$ is a graph of order $n$ with $\delta(G) \geq(n+1) / 2$, then $G$ is fully cycle extendable.

Here a graph $G$ is called fully cycle extendable if every vertex of $G$ lies on a triangle of $G$ and furthermore every nonhamiltonian cycle $C$ of $G$ can be extended to another cycle $C^{\prime}$ such that $V(C) \subseteq V\left(C^{\prime}\right)$ and $\left|V\left(C^{\prime}\right)\right|=|V(C)|+1$. Hence, if a graph is fully cycle extendable then it is surely pancyclic. By Lemma 1, if $\delta(G) \geq(n+1) / 2$, then $G$ is pancyclic. So we only need to consider graphs whose minimum degree is less than $(n+1) / 2$. Suppose that $G$ is a graph of order $n$ satisfying the conditions of Theorem 11 and with $\delta(G)<(n+1) / 2$, i.e., 
$\delta(G) \leq n / 2$. Let $S$ be a minimal cut set of $G$. Since $\delta(G) \leq n / 2,|S| \leq n / 2$. The following claim was given by $\mathrm{Li}$ et al. in [16]. We add its proof for convenience.

Claim 1 (Li et al. [16]). Every vertex of $S$ is adjacent to every vertex of $V(G) \backslash S$.

Proof Clearly, the choice of $S$ implies that for every vertex $x \in S$ and every component $H$ of $G-S, x$ is adjacent to at least one vertex of $H$; otherwise $S \backslash\{x\}$ is a vertex cut, contradicting the choice of $S$. Suppose that $x y \notin E(G)$ for some $y \in V(G) \backslash S$. Let $H$ be the component of $G-S$ containing $y$, let $P$ be a shortest path from $y$ to $x$ with all internal vertices in $H$, and let $y^{\prime}$ be a neighbor of $x$ in a component of $G-S$ other than $H$. Then $y P x y^{\prime}$ is an induced path on at least 4 vertices, contradicting that $G$ is $P_{4}$-free.

We now proceed with the proof of Theorem 11 and consider two cases.

Case 1. $|S|=n / 2$.

Clearly, $n$ is even and $\mid V(G) \backslash S) \mid=n / 2$. Denote $S=\left\{x_{1}, x_{2}, \ldots, x_{n / 2}\right\}$, $V(G) \backslash S=\left\{y_{1}, y_{2}, \ldots, y_{n / 2}\right\}$. If $S$ and $V(G) \backslash S$ are independent sets, then $G=K_{n / 2, n / 2}$, and we get the result. Without loss of generality, assume that $S$ is not independent and $x_{1} x_{2} \in E(S)$. By Claim $1, K_{n / 2, n / 2}$ is a spanning subgraph of $G$. Then from $K_{n / 2, n / 2}$ we can get all cycles of even length from 4 up to $n-2$ containing $x_{1}$ but not $x_{2}$. By inserting $x_{2}$ after vertex $x_{1}$ in every even cycle, we get all cycles of odd length from 5 up to $n-1$. Since $x_{1} x_{2} y_{1} x_{1}$ is a 3-cycle and $K_{n / 2, n / 2}$ contains an $n$-cycle, $G$ is pancyclic.

Case 2. $|S|<n / 2$.

Let $s=|S|$, hence $|V(G) \backslash S|=n-s$, and clearly $n-s>n / 2$. Since $G$ is 1 tough, $G-S$ is not independent. Let $H$ be the subgraph of $G$ induced by $S$ and $s$ vertices of $G-S$ that contain at least one adjacent pair. Then $K_{s, S}$ is a spanning subgraph of $H$. By the same method as in Case 1, we can prove that $H$ contains cycles of length 3 up to $2 s$, and hence $G$ contains cycles of length 3 up to $2 s$.

We know from earlier results that every 1-tough $P_{4}$-free graph on at least three vertices is hamiltonian, so $G$ contains a Hamilton cycle $C$. Let the vertices of $S=\left\{x_{1}, x_{2}, \ldots, x_{s}\right\}$ be arranged in this order around $C$ according to a fixed orientation of $C$, and denote every segment of $C$ from $x_{i}$ to $x_{i+1}$ by $S_{i}=$ $x_{i} y_{i_{1}} y_{i_{2}} \ldots y_{i_{r_{i}}} x_{i+1}$ (possible with $r_{i}=0$, i.e., no vertex $y_{i}$ in between for some $1 \leq i \leq s)$. By Claim $1, x_{i}$ is adjacent to every vertex of $S_{i} \backslash\left\{x_{i+1}\right\}$. Hence, if $r_{i} \neq 0$, then we can get cycles of length from $n$ down to $n-r_{i}+1$ using $x_{i} y_{i_{k}} C x_{i}$ $\left(1 \leq k \leq r_{i}\right)$. In this way, if $r_{i} \neq 0$ for each $1 \leq i \leq s$, then we can delete the vertices within every segment one by one, until we are left with only two end vertices and one inside. Thus, we get cycles with length from $n$ down to $2 s$. Hence, $G$ is pancyclic. If $r_{i}=0$ for some $1 \leq i \leq s$, we can use similar arguments for the segments with $r_{i} \neq 0$ to get cycles with all possible missing lengths. Hence, $G$ is pancyclic. This completes the proof of Theorem 11. 


\section{Proof of Theorem 13}

One direction of the equivalence statement in Theorem 13 follows directly from Theorems 9-11, whereas the graphs $\mathcal{C}_{5}^{s}$ show that forbidding the induced subgraph $K_{1} \cup P_{4}$ does not imply the graphs are pancyclic. Hence, it suffices to prove that there is no graph $H$, apart from the proper induced subgraphs of $K_{1} \cup P_{4}$, that can ensure every $H$-free graph with toughness larger than one on at least three vertices is pancyclic. We use the following lemma to complete our proof.

Lemma 2 (Li et al. [16]) Let $R$ be a graph on at least three vertices. If $R$ is not an induced subgraph of $K_{1} \cup P_{4}$, then $R$ contains one of the graphs in $\mathcal{H}=$ $\left\{C_{3}, C_{4}, C_{5}, K_{1,3}, 2 K_{2}, 4 K_{1}\right\}$ as an induced subgraph.

Using Lemma 2, it is sufficient to prove that for each of the graphs $R \in \mathcal{H}$ not every $R$-free graph on at least three vertices with toughness larger than one is pancyclic. To show this, we give suitable counterexamples for each case. Most of the counterexamples we present here also appear in [23], in which they serve as counterexamples for hamiltonian-connectivity. For the class of $4 K_{1}$-free graphs, the small graph sketched in Fig. 3 is $4 K_{1}$-free and has toughness larger than one, but is not pancyclic. For counterexamples related to the other members of the class of graphs $\mathcal{H}$ we refer to some useful known results.

For $R=C_{3}$, the Petersen graph is a suitable counterexample, since it is $C_{3}$-free, has toughness $4 / 3$ and is nonhamiltonian.

For $R=C_{4}, C_{5}$ or $2 K_{2}$, we can find suitable split graphs as counterexamples. Split graphs are known to be $\left\{C_{4}, C_{5}, 2 K_{2}\right\}$-free. It was proved in [15] that every $\frac{3}{2}$ tough split graph is hamiltonian, and that there is a sequence $\left\{G_{n}\right\}_{n=1}^{\infty}$ of split graphs with no 2-factor (a 2-regular spanning subgraph, not necessarily connected) and $\tau\left(G_{n}\right) \rightarrow 3 / 2$. Here, we select the latter graphs to serve as examples for our purposes.

For $R=K_{1,3}$, every claw-free noncomplete graph $G$ has the property that $2 \tau(G)=\kappa(G)$, where $\kappa(G)$ denotes the (vertex) connectivity of $G$. In [17], it is conjectured that every 4-connected claw-free graph is hamiltonian, and the authors give examples of 3-connected (hence 3/2-tough) claw-free graphs that are not hamiltonian. These examples clearly serve our purposes and complete our proof of Theorem 13.

Fig. $34 K_{1}$-free non-pancyclic graph

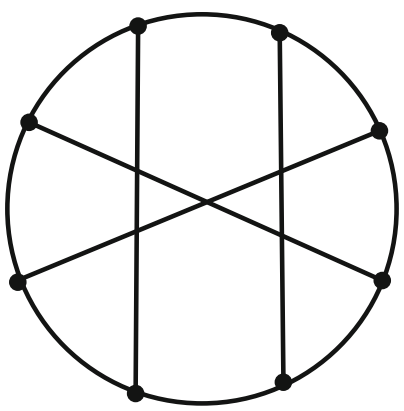




\section{Compliance with Ethical Standards}

Conflict of Interest The authors declare that they have no conflict of interest.

Open Access This article is licensed under a Creative Commons Attribution 4.0 International License, which permits use, sharing, adaptation, distribution and reproduction in any medium or format, as long as you give appropriate credit to the original author(s) and the source, provide a link to the Creative Commons licence, and indicate if changes were made. The images or other third party material in this article are included in the article's Creative Commons licence, unless indicated otherwise in a credit line to the material. If material is not included in the article's Creative Commons licence and your intended use is not permitted by statutory regulation or exceeds the permitted use, you will need to obtain permission directly from the copyright holder. To view a copy of this licence, visit http:// creativecommons.org/licenses/by/4.0/.

\section{References}

1. Bauer, D., Broersma, H.J., van den Heuvel, J.P.M., Veldman, H.J.: On Hamiltonian properties of 2-tough graphs. J. Graph Theory. 18, 539-543 (1994)

2. Bauer, D., Broersma, H.J., Veldman, H.J.: Not every 2-tough graph is hamiltonian. Discret. Appl. Math. 99, 317-321 (2000)

3. Bauer, D., Broersma, H.J., Schmeichel, E.: Toughness in graphs-a survey. Graphs Comb. 22, 1-35 (2006)

4. Bedrossian, P.: Forbidden subgraph and minimum degree conditions for hamiltonicity. Thesis, Memphis State University, USA (1991)

5. Bondy, J.A.: Pancyclic graphs. In: Proceedings of the Second Louisiana Conference on Combinatorics, Graph Theory and Computing, Louisiana State University, Baton Rouge, LA, pp. 167-172 (1971)

6. Bondy, J.A., Murty, U.S.R.: Graph Theory. Springer Graduate Texts in Mathematics, vol. 244 (2008) ISBN-13: 978-1849966900

7. Brandt, S.: Sufficient conditions for graphs to contain all subgraphs of a given type. Ph.D. thesis, Freie Universität Berlin (1994)

8. Brause, C., Rautenbach, D., Schiermeyer, I.: Local connectivity, local degree conditions, some forbidden induced subgraphs, and cycle extendability. Discret. Math. 340, 596-606 (2017)

9. Broersma, H.: How tough is toughness?. Bulletin Eur. Assoc. Theor. Comput. Sci. EATCS 3(117) (2015)

10. Broersma, H.J., Patel, V., Pyatkin, A.: On toughness and hamiltonicity of $2 K_{2}$-free graphs. J. Graph Theory. 75, 244-255 (2014)

11. Chvátal, V.: Tough graphs and Hamiltonian circuits. Discret. Math. 5, 215-228 (1973)

12. Faudree, R.J., Gould, R.J.: Characterizing forbidden pairs for hamiltonian properties. Discret. Math. 173, 45-60 (1997)

13. Hendry, G.R.T.: Extending cycles in graphs. Discret. Math. 85, 59-72 (1990)

14. Jung, H.A.: On a class of posets and the corresponding comparability graphs. J. Comb. Theory Ser. B. 24, 125-133 (1978)

15. Kratsch, D., Lehel, J., Müller, H.: Toughness, hamiltonicity and split graphs. Discret. Math. 150, 231-245 (1996)

16. Li, B., Broersma, H.J., Zhang, S.: Forbidden subgraphs for hamiltonicity of 1-tough graphs. Discuss. Math. Graph Theory. 36, 915-929 (2016)

17. Matthews, M.M., Sumner, D.P.: Hamiltonian results in $K_{1,3}$-free graphs. J. Graph Theory 8, 139-146 (1984)

18. Nikoghosyan, Z.G.: Disconnected forbidden subgraphs, toughness and Hamilton cycles. ISRN Comb. (2013). https://doi.org/10.1155/2013/673971. (Article ID 673971)

19. Schiermeyer, I., Sonntag, M., Teichert, H.M.: Structural properties and hamiltonicity of neighborhood graphs. Graphs Comb. 26, 433-456 (2010)

20. Wang, J.L., Mou, L.: The hamiltonicity of $k$-connected $[s, t]$-graphs. Ars Comb. 118, 407-418 (2015)

21. Wang, J.L., You, H.Y.: Path extendability and degree sum in graphs. Ars Comb. 122, 181-192 (2015) 
22. Yue, J., Song, J.M.: Note on the perfect Roman domination number of graphs. Appl. Math. Comput. 364, 124685 (2020). https://doi.org/10.1016/j.amc.2019.124685 (ISSN 0096-3003)

23. Zheng, W., Broersma, H.J., Wang, L.G.: Toughness, forbidden subgraphs, and hamiltonian-connected graphs. Discuss. Math. Graph Theory (2019). https://doi.org/10.7151/dmgt.2247

Publisher's Note Springer Nature remains neutral with regard to jurisdictional claims in published maps and institutional affiliations.

\section{Authors and Affiliations}

\section{Wei Zheng ${ }^{1,2,3} \cdot$ Hajo Broersma ${ }^{2}$ (1) $\cdot$ Ligong Wang $^{3}$}

$\triangle$ Hajo Broersma

h.j.broersma@utwente.nl

Wei Zheng

zhengweimath@163.com

Ligong Wang

lgwangmath@163.com

1 School of Mathematics and Statistics, Shandong Normal University, Jinan 250358, Shandong, China

2 Faculty of EEMCS, University of Twente, P.O. Box 217, 7500 AE Enschede, The Netherlands

3 Department of Applied Mathematics, School of Science, Northwestern Polytechnical University, Xi' an 710072, Shaanxi, China 